\title{
The connection between gamma-ray bursts and supernovae lb/c
}

\author{
E. Bissaldi ${ }^{1, \star}$, F. Calura $^{2}$, F. Matteucci ${ }^{2,3}$, F. Longo ${ }^{1,4}$, and G. Barbiellini ${ }^{1,4}$ \\ 1 Dipartimento di Fisica, Universitá di Trieste, via Valerio 2, 34127, Trieste, Italy \\ e-mail: ebs@mpe.mpg.de \\ 2 INAF - Osservatorio Astronomico di Trieste, via G. B. Tiepolo 11, 34131 Trieste, Italy \\ 3 Dipartimento di Astronomia - Universitá di Trieste, via G. B. Tiepolo 11, 34131 Trieste, Italy \\ ${ }^{4}$ INFN, Sezione di Trieste, via Valerio 2, 34127 Trieste, Italy
}

Received 15 November 2006 / Accepted 21 February 2007

\begin{abstract}
Context. It has been established that Gamma-Ray Bursts (GRBs) are connected to Supernovae (SNe) explosions of type Ib/c. Aims. We intend to test whether the hypothesis of type $\mathrm{Ib} / \mathrm{c}$ SNe from different massive progenitors can reproduce the local GRB rate as well as the GRB rate as a function of redshift. We aim to predict the GRB rate at very high redshift under different assumptions about galaxy formation and star formation histories in galaxies.

Methods. We assume different star formation histories in galaxies of different morphological types: ellipticals, spirals and irregulars, which have already been tested in self-consistent galaxy models reproducing both chemical and photometrical properties of galaxies. We explore different hypotheses concerning the progenitors of type Ib/c SNe: i) single massive stars ( $M>25 M_{\odot}$, Wolf-Rayet stars), ii) massive close binaries (12-20 $M_{\odot}$ ), and iii) both Wolf-Rayet stars and massive binaries. We conclude that the mixed scenario (iii) is preferable to reproduce the local type $\mathrm{Ib} / \mathrm{c} \mathrm{SN}$ rates in galaxies and we adopt this scenario for comparison with the GRB rates.

Results. We find an excellent agreement between the observed GRB local rate and the predicted type Ib/c SN rate in irregular galaxies, when a range for single Wolf-Rayet stars of $40-100 M_{\odot}$ is adopted. We also predict the cosmic type $\mathrm{Ib} / \mathrm{c} \mathrm{SN}$ rate by taking into account all the galaxy types in a unitary volume of the Universe and we compare it with the observed cosmic GRB rate as a function of redshift. By assuming the formation of spheroids at high redshift, we predict a cosmic type Ib/c SN rate, which is always higher than the GRB rate, suggesting that only a small fraction $(0.1-1 \%)$ of type Ib/c SNe become GRBs. In particular, we find a ratio between the cosmic $\mathrm{GRB}$ rate and the cosmic type $\mathrm{Ib} / \mathrm{c}$ rate in the range $10^{-2}-10^{-3}$, in agreement with previous estimates. Finally, due to the high rate of star formation in spheroids at high redshift, which is our preferred scenario for galaxy formation, we predict more GRBs at high redshift than in the hierarchical scenario for galaxy formation, a prediction which remains to be proven by future observations.
\end{abstract}

Key words. galaxies: evolution - galaxies: fundamental parameters - stars: supernovae: general - gamma rays: bursts

\section{Introduction}

Gamma Ray Bursts (GRBs) are sudden and powerful flashes of gamma-ray radiation, occurring randomly at a rate of $\sim 1$ per day throughout the Universe. The duration of GRBs at MeV energies ranges from $10^{-3} \mathrm{~s}$ to about $10^{3} \mathrm{~s}$, with long bursts being characterized by a duration $>2 \mathrm{~s}$.

During the last few years, it has been established that at least a large fraction of long-duration GRBs is directly connected with the death of massive stars. This scenario is strongly supported by observations of supernova (SN) features in the spectra of several of GRB afterglows. Examples of the spectroscopic SN-GRB connection include SN 1998bw/GRB 980425 (Galama et al. 1998), SN 2003dh/GRB 030329 (Stanek et al. 2003; Hjorth et al. 2003), SN 20031w/GRB 031203 (Malesani et al. 2004), and SN 2006aj/GRB 060218 (Masetti et al. 2006; Campana et al. 2006, Modjaz et al. 2006).

Originally, $\mathrm{SNe}$ were classified into two main types (I, II) on the basis of the presence (type II) or absence (type I) of $\mathrm{H}$ lines in their spectra (Zwicky 1938). At the present time we distinguish among five $\mathrm{SN}$ types on the basis of the spectra obtained at maximum light. Classical type II SNe have prominent Balmer lines exhibiting P-Cygni profiles and represent about $70 \%$ of the

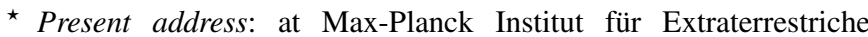
Physik, Giessenbachstraße 1, 85748 Garching, Germany. exploding stars in the Universe (Cappellaro et al. 1999). The classification can be further subdivided into types II-L and II-P, according to the shape of the light curve which can be linear (L) or have a plateau (P). Type IIn $\mathrm{SNe}$ have strong $\mathrm{H}$ lines in emission. They can be distinguished from the classical type II SNe by the lack of absorption in their Balmer lines. Chugai (1997) introduced this designation to reflect the fact that these $\mathrm{SNe}$ undergo significant interaction with a "dense wind" produced by the SN progenitor prior to explosion. Type Ia SNe are characterized by the lack of $\mathrm{H}$ lines and by a strong absorption observed at $\lambda \lambda 6347,6371 \AA$ attributed to the P-Cyg profile of Si II. Type Ib $\mathrm{SNe}$ are identified by spectra with no evident Balmer lines, weak or absent Si II lines and strong He I lines. Bertola (1964) reported the first observation of this class of $\mathrm{SNe}$, but the "Ib" designation was introduced later by Elias et al. (1985). Type Ic $\mathrm{SNe}$ are characterized by weak or absent $\mathrm{H}$ and $\mathrm{He}$ lines and no evident Si II. They show Ca II H\&K in absorption, the Ca II near-IR triplet with a P-Cygni profile, and O I in absorption. The "Ic" class was introduced by Wheeler \& Harkness (1986).

Interestingly, the $\mathrm{SN}$ features observed in the afterglows of GRBs resemble those of type Ib/c SNe (Della Valle 2006; Woosley \& Heger 2006, and references therein). In particular, modeling of the SN lightcurves reveals that the $\mathrm{SNe}$ with GRB-connection have very large explosion energy and mass production of ${ }^{56} \mathrm{Ni}$ compared to normal type $\mathrm{Ib} / \mathrm{c} \mathrm{SNe}$ 
(Iwamoto et al. 1998; Nakamura et al. 2001; Deng et al. 2005; Mazzali et al. 2006b), except SN 2006aj which requires an explosion energy that is comparable to that of normal $\mathrm{SNe} \mathrm{Ib/c}$ (Mazzali et al. 2006a). These facts have motivated people to invent the term "hypernovae" for this special and much more powerful class of SNe (Iwamoto et al. 1998; see also Paczyǹski 1998).

These phenomenological results greatly support the "collapsar" model (MacFayden \& Woosley 1999; Zhang et al. 2003) in which a Wolf-Rayet progenitor undergoes core collapse, producing a rapidly rotating black hole surrounded by an accretion disk which injects energy into the system and thus acts as a "central engine". The energy extracted from this system supports a quasi-spherical type $\mathrm{Ib} / \mathrm{c} \mathrm{SN}$ explosion and drives collimated jets through the stellar rotation axis which produce the prompt gamma-ray and afterglow emission (e.g., Zhang \& Mészáros 2004).

From the observations mentioned above, it is clear that a comparison between the type $\mathrm{Ib} / \mathrm{c} \mathrm{SN}$ rates in galaxies and the GRB rate is very important in order to test if the SN-GRB connection reflects what is currently known about $\mathrm{SN}$ progenitors, stellar and galactic evolution.

Galaxies of different morphological types evolve in different ways with the main determining factor of their evolution being the star formation history. Recently, Calura \& Matteucci (2006) calculated the rates of type II, Ib, and Ic SNe in galaxies of different morphological types (ellipticals, spirals and irregulars), by means of self-consistent galaxy evolution models, reproducing both the chemical and spectro-photometric properties of galaxies.

In this paper, we present a study of models which accurately reproduce the local type $\mathrm{Ib} / \mathrm{c} \mathrm{SN}$ rate in galaxies, by following the approach of Calura \& Matteucci (2006). We assume different progenitors for the type $\mathrm{Ib} / \mathrm{c} \mathrm{SNe}$ such as single Wolf-Rayet (WR) stars and massive stars $\left(12-20 M_{\odot}\right)$ in close binary systems, as well as several star formation histories typical of galaxies of different morphological types.

The assumed range for the binary progenitors of type $\mathrm{Ib} / \mathrm{c}$ $\mathrm{SNe}$ are based on suggestions by Baron (1992), Nomoto et al. (1996) and on the recent modelling of the proto-type Ic supernova SN 1994 I (Sauer et al. 2006), which suggests that the upper mass limit for these binary stars should not be larger than $20 M_{\odot}$. When considering the single WR stars as type Ib/c progenitors, we need to assume a lower mass limit for the formation of WR stars, but this limit is uncertain and strongly depends on the details of stellar evolution models, such as rotation and assumed mass loss, which in turn depend on the initial stellar chemical composition (see Meynet \& Maeder 2005 and Yoon et al. 2006 for a recent study on the single WR stars and GRBs). Therefore, we will explore several cases where the lower mass for the formation of a single WR star varies from 25 up $90 M_{\odot}$. However, it is important to recall that most stars with $M>25 M_{\odot}$ probably produce black holes, either in a prompt collapse or by fallback; in neither case would one expect a normal type $\mathrm{Ib} / \mathrm{c} \mathrm{SN}$, since probably most of the mass in the Si shell, which is turned into $\mathrm{Ni}$ during the explosion, would fall into the black hole. So, it is still unproven that massive $\mathrm{SNe}$ forming black holes would appear as typical $\mathrm{SNe} \mathrm{Ib} / \mathrm{c}$. If, instead, such massive stars are in interacting binary systems, their final core evolution is drastically changed and they are expected to produce neutron stars rather than black holes (Brown et al. 2001) and hence possibly become $\mathrm{SNe} \mathrm{Ib} / \mathrm{c}$. All these facts, seem to suggest that the binary channel for the formation of $\mathrm{SNe} \mathrm{Ib} / \mathrm{c}$ is favored over the channel of very massive single stars.
We then derive the SN rates and compare them with the observed local GRB rates, thus obtaining an estimate of the local $\mathrm{GRB} / \mathrm{SN}$ ratio. Furthermore, we move on cosmological scales by computing the cosmic type $\mathrm{Ib} / \mathrm{c} \mathrm{SN}$ rates on the basis of which we choose the best model for the GRB distribution as a function of redshift and therefore we predict the expected number of GRBs at high redshift.

The paper is organized as follows: in Sect. 2 we present the galactic models and the theoretical type Ib/c SN rates for galaxies of different morphological types. In Sect. 3 we discuss the local GRB rate and compare it with the theoretical predictions. In Sect. 4 we describe how we compute the cosmic type Ib/c SN rates and we extract a best model for the distribution of GRB as a function of redshift. Finally in Sect. 5 we draw some conclusions.

\subsubsection{Evolution models for galaxies}

In order to compute the $\mathrm{SN} \mathrm{Ib/c} \mathrm{rates} \mathrm{and} \mathrm{compare} \mathrm{them} \mathrm{with} \mathrm{the}$ observed GRB rate we adopted self-consistent galaxy evolutionary models already reproducing the majority of the properties of galaxies of different morphological types. In particular, these models are tuned to reproduce the present time SN rates and the chemical abundances in stars and gas. Moreover, the predicted star formation and chemical enrichment histories, when adopted in spectro-photometric models, are il line with photometric properties of galaxies.

In order to better understand the adopted galaxy models, a schematic outline of their basic equations and physical processes is provided (Calura \& Matteucci 2003).

Let $G_{i}(t)$ be the normalized fractional mass of gas within a galaxy in the form of the element $i$ :

$G_{i}(t)=\frac{M_{\mathrm{g}}(t) X_{i}(t)}{M_{\mathrm{tot}}}$

where $M_{\text {tot }}$ and $M_{\mathrm{g}}(t)$ are the total galaxy mass and the mass of gas at time $t$, respectively.

$X_{i}(t)=\frac{G_{i}(t)}{G(t)}$

represents the abundance in mass of the element $i$, the summation over all elements in the gas mixture being equal to unity. Thus, the quantity

$G(t)=\frac{M_{\mathrm{g}}(t)}{M_{\mathrm{tot}}}$

is the total fractional mass of gas present in the galaxy at time $t$.

The temporal evolution of $G_{i}(t)$ is described by the basic equation:

$\dot{G}_{i}=-\psi(t) X_{i}(t)+R_{i}(t)+\left(\dot{G}_{i}\right)_{\mathrm{inf}}-\left(\dot{G}_{i}\right)_{\mathrm{out}}$.

Here, $\psi(t)$ is the star formation rate (SFR), namely the fractional amount of gas turning into stars per unit time. $R_{i}(t)$ represents the returned fraction of matter in the form of an element $i$ that the stars eject into the interstellar medium (ISM) through stellar winds and SN explosions. This term contains all the prescriptions regarding the stellar yields and the SN progenitor models. The two terms $\left(\dot{G}_{i}\right)_{\text {inf }}$ and $\left(\dot{G}_{i}\right)_{\text {out }}$ account for the infalling external gas from the intergalactic medium and for the outflow, occurring by means of SN driven galactic winds, respectively.

Here we are interested in computing the $\mathrm{SN}$ rates in the framework of these self-consistent galaxy models. The two key 
ingredients for computing the SN rates are the SFR and the stellar initial mass function (IMF). The first is generally expressed as a function of time only, while the second, which describes the stellar mass distribution at birth, is likely to be universal (Kroupa 2002) and not vary as a function of time (Chiappini et al. 2000).

\subsubsection{Star formation rates}

The main feature characterizing a particular morphological galactic type is represented by the prescription adopted for the star formation history, summarized in the SFR expression.

In the case of elliptical galaxies, the SFR $\psi(t)$ (in $\mathrm{Gyr}^{-1}$ ) has a simple form and is given by:

$\psi(t)=v G(t)$.

The quantity $v$ is the efficiency of star formation, namely the inverse of the typical time scale for star formation.

In the case of ellipticals, $v$ is assumed to drop to zero at the onset of a galactic wind, which develops as the thermal energy of the gas heated by supernova explosions exceeds the binding energy of the gas (Arimoto \& Yoshii 1987; Matteucci \& Tornambé 1987). The estimated quantity is strongly influenced by assumptions concerning the presence and distribution of dark matter (Matteucci 1992); for the model adopted here a diffuse $\left(R_{\mathrm{e}} / R_{\mathrm{d}}=0.1\right.$, where $R_{\mathrm{e}}$ is the effective radius of the galaxy and $R_{\mathrm{d}}$ is the radius of the dark matter core) but massive $\left(M_{\text {dark }} / M_{\text {Lum }}=10\right)$ dark halo has been assumed.

In the case of irregular galaxies, a continuous star formation rate is assumed as in Eq. (5), but characterized by a lower efficiency than the one adopted for ellipticals.

In the case of spiral galaxies, the SFR expression is slightly more complex, since it takes into account the feedback between SNe and ISM by means of a dependence upon the total surface mass density (see Chiosi 1980; Chiappini et al. 1997):

$\psi(r, t)=v\left[\frac{\sigma(r, t)}{\sigma\left(r_{\odot}, t\right)}\right]^{2(k-1)}\left[\frac{\sigma\left(r, t_{\mathrm{Gal}}\right)}{\sigma(r, t)}\right]^{k-1} G^{k}(r, t)$,

where $v$ is the SF efficiency, $\sigma(r, t)$ is the total (gas + stars) surface mass density at a radius $r$ and time $t$, and $\sigma\left(r_{\odot}, t\right)$ is the total surface mass density in the solar region. For the gas density exponent $k$ a value of 1.5 has been assumed by Chiappini et al. (1997) in order to ensure a good fit with the observational constraints at the solar vicinity and in agreement with the estimates by Kennicutt (1998).

The three different star formation rates for elliptical, irregular and spiral galaxy models as functions of time are shown in Fig. 1. The SFR of the elliptical model (corresponding to a galaxy with luminous mass of $10^{11} M_{\odot}$ ) is characterized by very high SFR values (from 100 to $1000 M_{\odot} \mathrm{yr}^{-1}$ ) and by a starburst lasting $\sim 0.2 \mathrm{Gyr}$, which traces the rapid collapse of a homogeneous sphere of primordial gas where star formation is taking place at the same time as the collapse proceeds. Star formation halts as the energy of the ISM, heated by stellar winds and SN explosions, balances the binding energy of the gas. At this time a galactic wind occurs, sweeping away almost all the residual gas.

The SFR of the irregular model is continuous and is characterized by a low SF efficiency. In this scenario, irregulars are assumed to assemble from merging of protogalactic small clouds of primordial chemical composition, until a mass of $\sim 6 \times 10^{9} M_{\odot}$ is accumulated, therefore producing stars at a lower rate than spiral and elliptical galaxies.

The predicted SFR for spirals is characterized by two peaks due to two infall episodes present in the model of

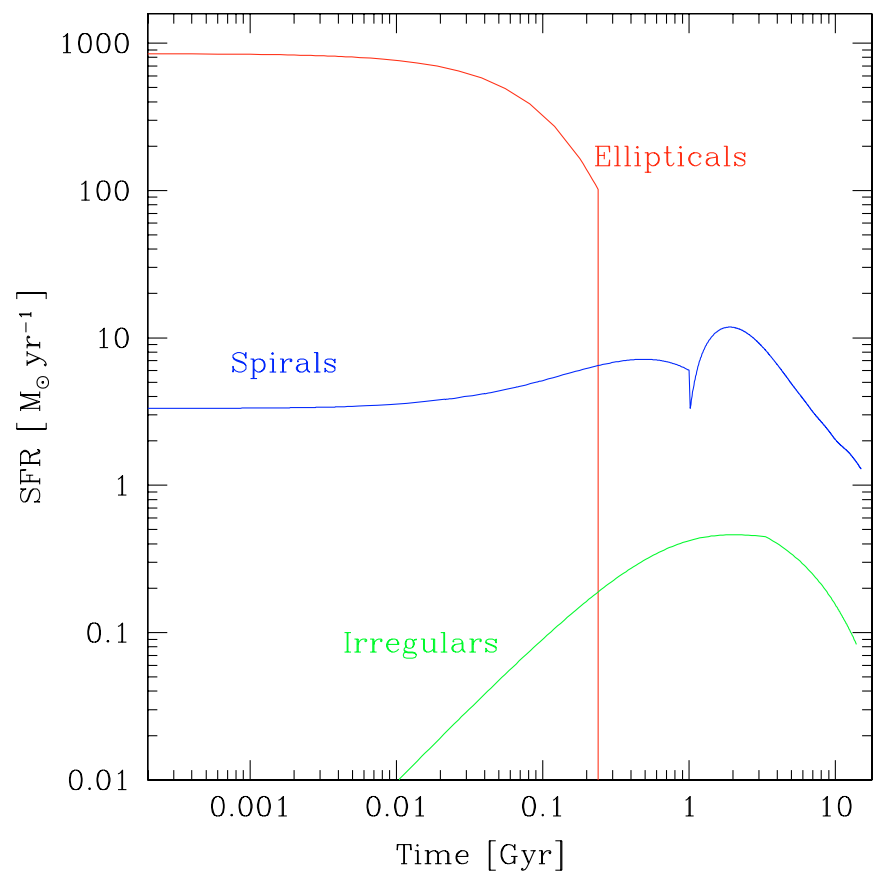

Fig. 1. Star formation rates, expressed in $M_{\odot} \mathrm{yr}^{-1}$, as functions of time calculated according to Eqs. (5) and (6) for elliptical (upper curve), spiral (median curve) and irregular (lower curve) galaxy models.

Chiappini et al. (1997). During the first episode, the halo forms and the gas shed by the halo rapidly gathers in the center, yielding the formation of the bulge. During the second episode, a slower infall of external gas forms the disk, with the gas accumulating faster in the inner than in the outer region ("inside-out" scenario; Matteucci \& François 1989). The process of disk formation is much longer than the halo and bulge formation, with timescales varying from $\sim 2 \mathrm{Gyr}$ in the inner disk to $\sim 7 \mathrm{Gyr}$ in the solar region and up to $15-20 \mathrm{Gyr}$ in the outermost disk. The two infall episodes are clearly visible in Fig. 1. The second one is dominated by the contribution of the disk. Here, the SFR for the MW represents an average between 2 and $20 \mathrm{kpc}$ from the galactic center, and it is assumed as representative for an average spiral galaxy.

\subsubsection{Initial mass function}

The most widely used functional form for the IMF is an extension of that proposed by Salpeter (1955) to the whole stellar mass range:

$\phi_{\text {Salpeter }}(M)=\mathcal{A}_{\text {Salpeter }} M^{-(1+x)}$,

where $x=1.35, \mathcal{A}_{\text {Salpeter }} \simeq 0.17$ is the normalization constant derived from:

$\int_{M_{\text {low }}}^{M_{\text {up }}} M \phi_{\text {Salpeter }}(M) \mathrm{d} M=1$.

The IMF in Eq. (7) is by number with $M_{\text {low }}=0.1 M_{\odot}$ and $M_{\text {low }}=100 M_{\odot}$. We are adopting this IMF for ellipticals and irregulars, where there is not a direct measure of the IMF. On the other hand, multi-slope expressions of the IMF give a better description of the luminosity function of the main sequence stars in the solar neighbourhood, as proposed by Tinsley (1980) and Scalo $(1986,1998)$. A simplified two-slope approximation to the actual Scalo (1986) IMF, that we adopt for the Milky Way and spirals, is given by:

$\phi_{\text {Scalo86 }}(M)=\mathcal{A}_{\text {Scalo86 }} M^{-(1+x)}$, 
where

$x=1.35 \mathcal{A}_{\text {Scalo86 }} \simeq 0.19$ if $M \leq 2 M_{\odot}$

$x=1.7 \mathcal{A}_{\text {Scalo86 }} \simeq 0.24$ if $M>2 M_{\odot}$.

Also for this IMF holds the normalization condition expressed in Eq. (8) for the mass range 0.1-100 $M_{\odot}$. Finally, one can consider the so-called "top-heavy" IMF, which was suggested by observational and theoretical arguments (e.g. Larson 1998 and references therein) and which assumes that the mass scale of the IMF was higher at earlier stages of the Universe, i.e. at high redshift:

$\phi_{t-h}(M)=\mathcal{A}_{t-h} M^{-(1+x)}$,

where $x=1.0$ and $\mathcal{A}_{t-h} \simeq 0.14$. However, this kind of IMF does not produce satisfactory results for the solar neighbourhood and it is better to assume a constant IMF (Chiappini et al. 2000).

\section{1. $S N R_{\mathrm{lb} / \mathrm{c}}$ models}

Generally, type Ib/c SNe occur in the vicinity of star forming regions and usual candidates for their progenitors are represented by massive WR stars (e.g. Hamuy 2003). However, the limiting mass for the formation of a WR star depends on the assumptions made in stellar models such as mass loss rate and rotation as recently shown by Meynet \& Maeder (2005). Their results suggest that the limiting mass for forming a WR star increases with decreasing metallicity because of the growth of the mass loss rate with metallicity. For the sake of simplicity we will start here by assuming that all massive stars with initial mass $M \gtrsim 25 M_{\odot}$ are becoming WR stars and explode as type Ib/c SNe. Then we will also test larger values for this mass limit.

The type $\mathrm{Ib} / \mathrm{c} \mathrm{SN}$ rate is then expressed as:

$$
\begin{aligned}
\operatorname{SNR}_{\mathrm{Ib} / \mathrm{c}}^{\text {Model I }}(t) & =\int_{25}^{100} \phi(M) \psi\left(t-\tau_{M}\right) \mathrm{d} M \\
& \simeq \psi(t) \int_{25}^{100} \phi(M) \mathrm{d} M,
\end{aligned}
$$

where $\tau_{M}$ is the stellar lifetime. Hereafter, Eq. (11) will be referred to as model I.

However, some observational evidence seems to go against the WR progenitor hypothesis. In particular, type Ib/c SNe can show broader light curves than expected assuming a massive star collapse origin. Other problems are represented by the apparent paucity of WR stars, unable to account for the rates observed in local galaxies (Muller et al. 1992), along with the fact that some events have been observed relatively far from active star forming regions (Filippenko \& Sargent 1991). More promising candidates, which could account for these observed differences, are represented by less massive stars, with typical masses of 12-20 $M_{\odot}$ (e.g. Baron 1992), in close binary systems. In this case, the loss of their envelope occurs by means of Roche-Lobe overflows. In this scenario (model II), the SN rate can be expressed as:

$$
\begin{aligned}
\operatorname{SNR}_{\mathrm{Ib} / \mathrm{c}}^{\text {Model II }}(t) & =F \int_{12}^{20} \phi(M) \psi\left(t-\tau_{M}\right) \mathrm{d} M \\
& \simeq F \psi(t) \int_{12}^{20} \phi(M) \mathrm{d} M,
\end{aligned}
$$

where $F$ is a parameter and represents the fraction of massive binary systems of that particular kind giving rise to type $\mathrm{Ib} / \mathrm{c}$

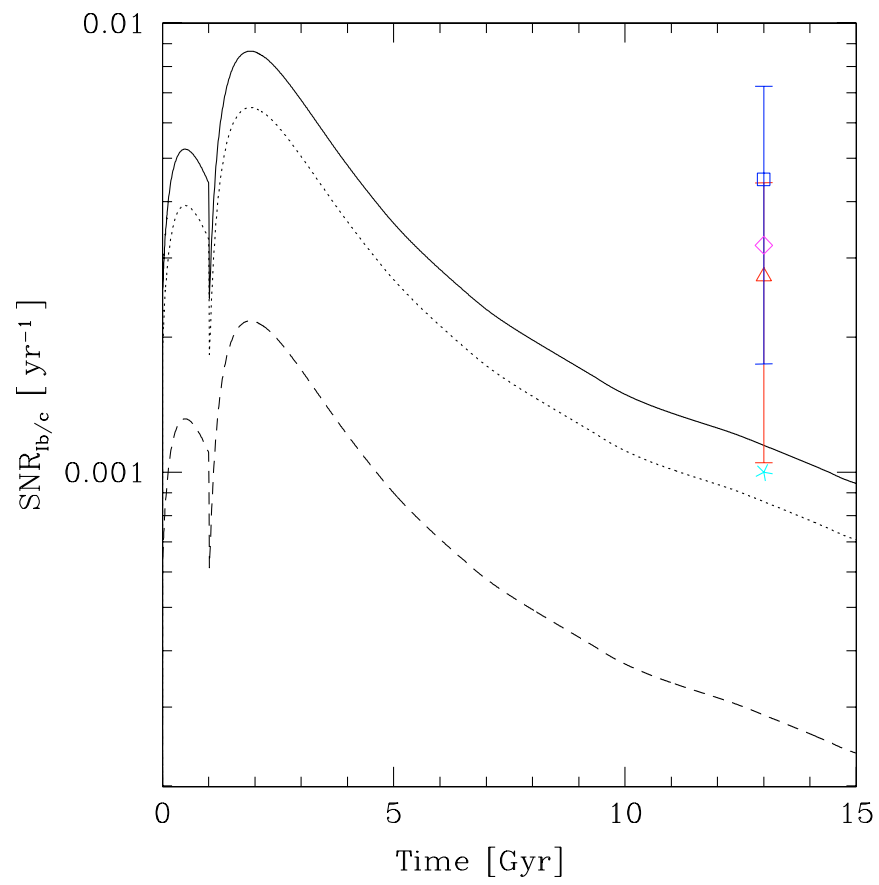

Fig. 2. SN rate for a spiral galaxy as a function of time calculated according to model I (Eq. (11), dotted line); model II (Eq. (12), dashed line); model III (Eq. (14), solid line). Data from Cappellaro et al. (1999) (open triangle), Mannucci et al. (2005) (open square), Della Valle (2005) (open rhombus) and Podsiadlowski et al. (2004) (star). A Scalo (1986) IMF is assumed.

SNe. This parameter is chosen to be equal to 0.15 (Calura \& Matteucci, 2006). This choice is motivated by the facts that first, in any galaxy, half of the massive stars are in binary systems, and second, that the fraction of massive stars in close binary systems (namely those which can give rise to $\mathrm{SNe} \mathrm{Ib} / \mathrm{c}$ ) can be reasonably estimated at $\sim 30 \%$, i.e. similar to the close binary frequency predicted for low mass systems (Jeffries \& Maxted 2005). Therefore the estimated value for this parameter is given by:

$F \simeq 0.5 \cdot 0.3 \simeq 0.15$.

This probably represents an inferior limit for the fraction of binary systems giving rise to $\mathrm{SNe} \mathrm{Ib} / \mathrm{c}$, as suggested in a paper by Podsiadlowski et al. (1992), where they concluded that $15-30 \%$ of all massive stars $\left(M>8 M_{\odot}\right)$ can produce $\mathrm{SNe} \mathrm{Ib}$. Moreover, a recent estimate by Kobulnicky et al. (2006) seems to favor the higher estimate. However, our choice of the binary fraction is also tuned to reproduce the observed $\mathrm{SN} \mathrm{Ib} / \mathrm{c}$ rates in galaxies, as shown by Calura \& Matteucci (2006), when single WR stars are also taken into account. Therefore, taking both scenarios into account, a cumulative $\mathrm{SN}$ rate is obtained (model III):

$$
\begin{aligned}
& \operatorname{SNR}_{\mathrm{Ib} / \mathrm{c}}^{\text {Model III }}(t)=\mathrm{SNR}_{\mathrm{Ib} / \mathrm{c}}^{\text {Model I }}(t)+\mathrm{SNR}_{\mathrm{Ib} / \mathrm{c}}^{\text {Model II }}(t) \\
& \simeq \psi(t)\left(F \int_{12}^{20} \Phi(M) \mathrm{d} M+\int_{25}^{100} \Phi(M) \mathrm{d} M\right) .
\end{aligned}
$$

In this case, the global fraction of type Ib/c SN progenitors is similar to the above fractions of massive binaries by Podsiadlowski et al. (1992). The three SN rates (models I, II and III) as functions of time in the local Universe, i.e. for a spiral galaxy like the Milky Way, are plotted in Fig. 2. A Scalo (1986) IMF (Eq. (9)) is assumed. The two main infall episodes, which are characteristic for the halo and for the disk formation, respectively, are clearly visible. 

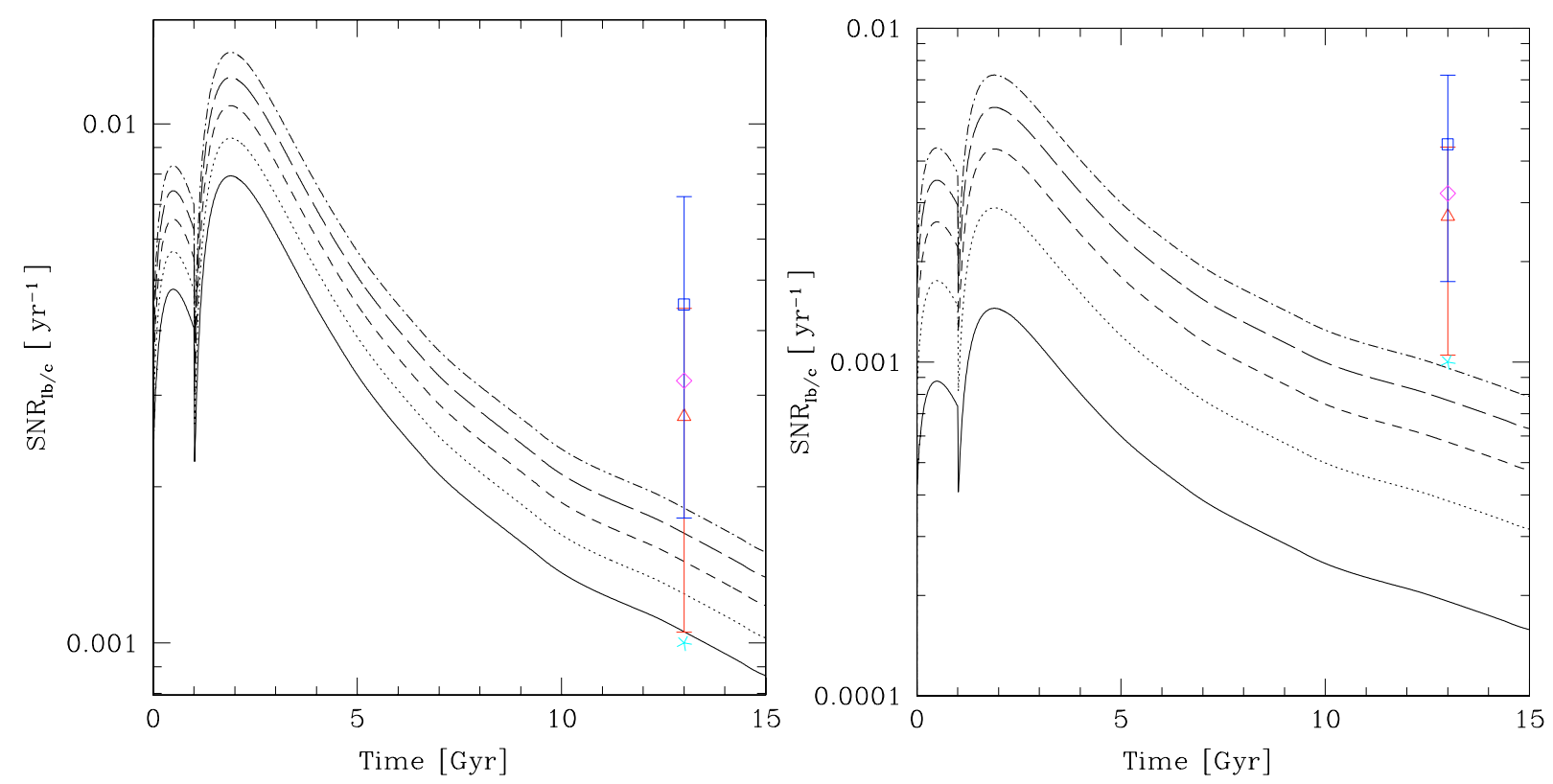

Fig. 3. SN rate for a spiral galaxy as a function of time calculated according to Model II (Eq. (12), left panel) and model III (Eq. (14), right panel). Different values of the parameter $F$ are considered: 0.1 (solid line); 0.2 (dotted line); 0.3 (short-dashed line); 0.4 (long-dashed line); 0.5 (dot-dashed-line). Data from Cappellaro et al. (1999) (open triangle), Mannucci et al. (2005) (open square), Della Valle (2005) (open rhombus) and Podsiadlowski et al. (2004) (star). A Scalo (1986) IMF is assumed in both cases.

Observational rates measured by different authors are also indicated at present time $(t \sim 13.47 \mathrm{Gyr})$. The $\mathrm{SN}$ rates are usually expressed in supernova units, SNus, $(1 \mathrm{SNu}=1 \mathrm{SN}$ per 100 yr per $10^{10} L_{B \odot} ; 1 \mathrm{SNuK}=1 \mathrm{SN}$ per $100 \mathrm{yr}$ per $10^{10} L_{K \odot}$ ). The rate of type $\mathrm{Ib} / \mathrm{c} \mathrm{SN}$ exploding in spiral galaxies, i.e. type $\mathrm{Sbc} / \mathrm{Sd}$, was measured by Cappellaro et al. (1999) obtaining:

$\mathrm{SNR}_{\mathrm{Ib} / \mathrm{c}}(t \sim 13.47 \mathrm{Gyr})=(0.14 \pm 0.07) \mathrm{SNu}$.

For the same rate, Mannucci et al. (2005) calculated a value:

$\operatorname{SNR}_{\mathrm{Ib} / \mathrm{c}}(t \sim 13.47 \mathrm{Gyr})=\left(0.067_{-0.032}^{+0.041}\right) \mathrm{SNuK}$.

In order to compare these rates with the $\mathrm{SN}$ rate models, which are calculated in units of $\mathrm{SNe} \mathrm{yr}^{-1}$, one needs to multiply them by the correct galactic luminosities. For spiral galaxies, the Milky Way $B$-band luminosity $L_{B}=(1.8 \pm 0.3) \times 10^{10} L_{B} \odot$ (van der Kruit 1986) and $K$-band luminosity $L_{K} \simeq 6.7 \times$ $10^{10} L_{K \odot}$ (Kent et al. 1991) are assumed.

Furthermore, the validity of the choice of the adopted massive binary star parameter, namely $F=0.15$, has been checked, performing a fine tuning with different values $0.1 \leq F \leq 0.5$ (Fig. 3), both for the rates calculated from model II (binary massive stars model) and model III (cumulative model). It is evident that $F=0.15$ represents quite a reasonable choice and it is in good agreement with the observations, especially for the binary massive stars model (model II). This is consistent with the results obtained by Calura \& Matteucci (2006).

Extending the analysis to elliptical and irregular galaxies, the results for the cumulative SN rate model are shown in Fig. 4. A Salpeter IMF has been adopted for modeling the SN rate in ellipticals and irregulars. $F=0.15$ is assumed. The observed rates are indicated for comparison in the correct units. In the case of irregular galaxies, the conversion from SNus is calculated by applying the $B$-band luminosity of the main body of the Small Magellanic Cloud (Vangioni-Flam et al. 1980), i.e. $L_{B}^{\text {irreg }} \simeq 7.8 \times 10^{8} L_{B \odot}$.

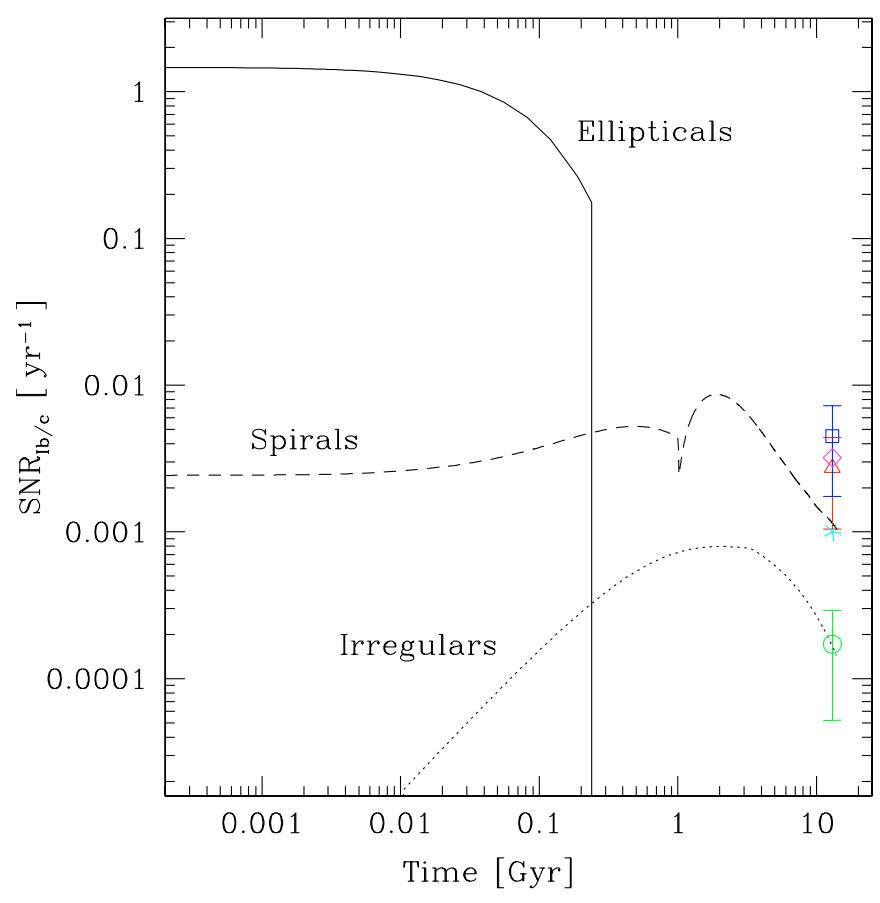

Fig. 4. Model III SN rate as a function of time for galaxies of different morphological types: Ellipticals (solid line), spirals (dashed line) and irregulars (dotted line). $F=0.15$ is assumed. Data from Cappellaro et al. (1999) (open triangle), Mannucci et al. (2005) (open square), Della Valle (2005) (open rhombus) and Podsiadlowski et al. (2004) (star); experimental data for the local SN rate in irregular galaxies (open circle) are also shown.

Therefore, the rate measured in irregulars by Cappellaro et al. (1999):

$\mathrm{SNR}_{\mathrm{Ib} / \mathrm{c}}(t \sim 13 \mathrm{Gyr})=(0.22 \pm 0.16) \mathrm{SNu}$,

is converted in to a value of:

$\operatorname{SNR}_{\mathrm{Ib} / \mathrm{c}}(t \sim 13 \mathrm{Gyr})=(1.72 \pm 1.2) \times 10^{-4} \mathrm{yr}^{-1}$. 


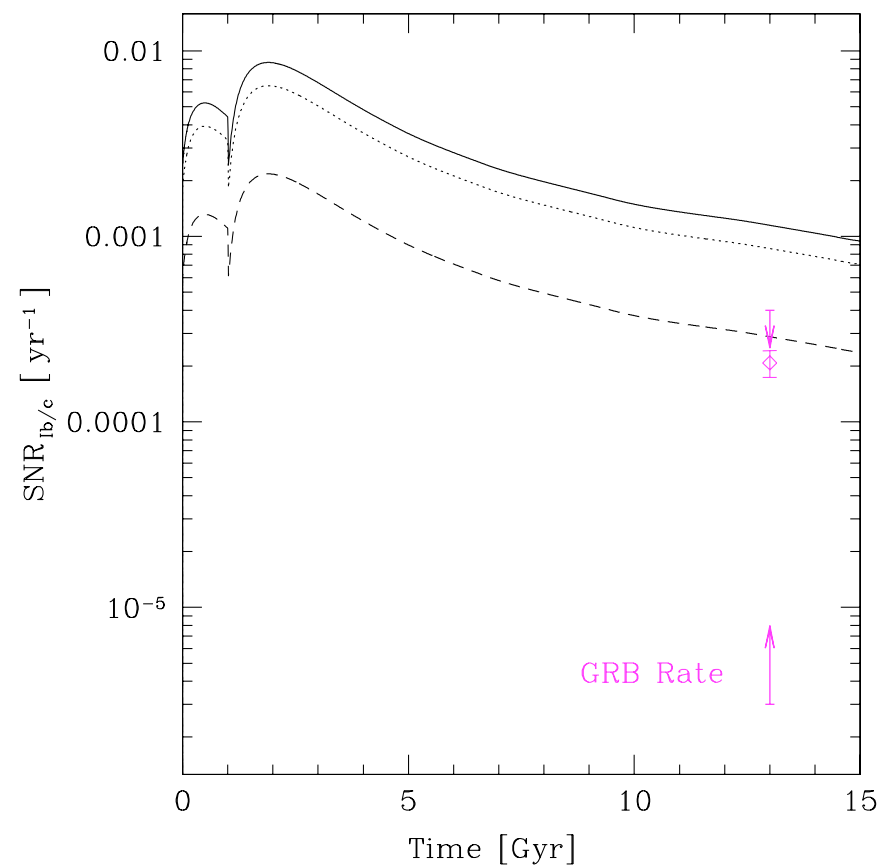

Fig. 5. SN rate for a spiral galaxy as a function of time calculated according to Model I (Eq. (11), dotted line); model II (Eq. (12), dashed line); model III (Eq. (14), solid line). A Scalo (1986) IMF and $F=0.15$ are assumed. Upper and lower limits for the observed local GRB rates (Eq. (16)) are over-plotted (upper and lower arrows). The open rhombus represents a "typical" value calculated from a local uncorrected rate by Zhang \& Mészáros (2004) and a beaming correction value by Frail et al. 2001 (see Eq. (17)).

From Fig. 4 it is evident that the SN rate model for an irregular galaxy reproduces this observed rate well.

\subsection{The local GRB rate}

The rate of observed GRBs in a galaxy such as the Milky Way can be established from the BATSE monitoring as $R_{\text {obs. }}$. Sokolov (2001) calculated this value assuming that all or at least most long GRBs were associated with $\mathrm{SNe} \mathrm{Ib} / \mathrm{c}$. From the observations of known GRB host galaxies between 1997 and 2000 (Bloom et al. 2002), he deduced that the absolute magnitude $M_{\text {HostGal }}$ ranged from 22 to $28.5 \mathrm{mag}$. The search for direct GRB-SN associations in nearby galaxies represents a challenge, because the majority of the SNe related to GRBs are faint (22-26 mag) and in very distant galaxies with $z \geq 0.4-$ 4.5. Sokolov (2001) considered the number of galaxies brighter than $26 \mathrm{mag}$ in one square degree of the sky, $N_{\mathrm{gal}}<26 \mathrm{mag} \approx$ $2 \times 10^{5}$ galaxy $\mathrm{deg}^{-2}$ (Casertano et al. 2000), and the number of GRBs observed by BATSE, $n_{\mathrm{GRB}} \approx 0.01 \mathrm{deg}^{-2} \mathrm{yr}^{-1}$. From these two quantities he calculated a rate of GRB events $R_{\text {obs }} \sim 5.0 \times 10^{-8}$ galaxy $^{-1} \mathrm{yr}^{-1}$. Other local values calculated by different authors are reported in Table 1 (left table) in units of GRB $\mathrm{yr}^{-1}$.

\subsection{1. "True" GRB rates}

The so-called true GRB rate is given by:

$R_{\mathrm{GRB}}=\left\langle f_{\mathrm{b}}^{-1}\right\rangle R_{\mathrm{obs}}$,

where the quantity $f_{\mathrm{b}}^{-1}$ is the beaming factor (Sari et al. 1999), which accounts for the fact that a GRB does not light up the full celestial sphere but rather a fraction. Since the possible corrections for the beaming factor cover a large range of values (see Table 1, right table), only plausible upper and lower limits can be estimated for the local GRB rate:

$$
\begin{aligned}
R_{\mathrm{GRB}, \min } & \simeq\left\langle f_{b, \min }^{-1}\right\rangle \cdot R_{\mathrm{obs}, \min } \\
& \sim 3 \times 10^{-6} \mathrm{yr}^{-1}, \\
R_{\mathrm{GRB}, \max } & \simeq\left\langle f_{b, \max }^{-1}\right\rangle \cdot R_{\mathrm{obs}, \max } \\
& \sim 4 \times 10^{-4} \mathrm{yr}^{-1},
\end{aligned}
$$

where only values calculated after 2004 were considered.

A "typical" value that is often considered in literature is given by the local rate calculated adopting the uncorrected value by Zhang \& Mészáros (2004) and the beaming factor by Frail et al. (2001), namely:

$$
\begin{aligned}
R_{\mathrm{GRB}} & \simeq(520 \pm 85) \cdot\left(4.0 \times 10^{-7}\right) \mathrm{yr}^{-1} \\
& \sim(2.08 \pm 0.34) \times 10^{-4} \mathrm{yr}^{-1}
\end{aligned}
$$

Figure 5 shows the distributions of the three SN rate Models (from Eqs. (11), (12) and (14)) for a spiral galaxy compared with the observed local GRB rates. Again, a Scalo (1986) IMF and $F=0.15$ are assumed for models I-III. The arrows mark the lower and upper limits for the GRB rates as calculated in Eq. (16), while the open rhombus represents the "typical" GRB rate calculated in Eq. (17).

As one can see from Fig. 5, the SN rate Models reproduce the observed local GRB rates poorly. However, uncertainties regarding type $\mathrm{Ib} / \mathrm{c} \mathrm{SN}$ progenitor masses allow us to perform a tuning of model I (massive single star model, see Eq. (11)). In particular, we considered different mass ranges for the progenitors of type Ib/c SNe, namely: $\Delta_{\mathrm{M} 1}=25-100 M_{\odot} ; \Delta_{\mathrm{M} 2}=40-100 M_{\odot}$; $\Delta_{\mathrm{M} 3}=60-100 M_{\odot} ; \Delta_{\mathrm{M} 4}=80-100 M_{\odot} ; \Delta_{\mathrm{M} 5}=90-100 M_{\odot}$. The behaviours of the SN rate calculated with model I and model III in spirals are then shown in Fig. 6 (left and right panel, respectively). This is allowed by the fact that the limiting mass for the formation of a WR star is still uncertain and depends on the stellar mass loss, which in turn depends on the stellar metallicity.

Taking a closer look at model I, it is evident that the range of observed GRB rates, in particular the one between the "typical" value (open circle) and the lower limit (bottom arrow), is better reproduced if $M_{\text {inf }} \gtrsim 40 M_{\odot}$ is assumed. This is consistent with the observations of the four GRBs with a clear SN association (see Introduction), which predict a progenitor mass as large as $40 M_{\odot}$ in three out of four cases.

Another interesting distribution to study is the $\mathrm{SN}$ rate for model III in galaxies of different morphological types together with the observed local GRB rates (Fig. 7). Again, a Salpeter IMF is adopted for ellipticals and irregulars. Here the integration is performed over the mass range $\Delta_{\mathrm{M} 2}=40-100 M_{\odot}$ for single massive stars giving rise to WRs. The parameter $F=0.15$, as previously discussed. It is evident that in all cases the $\mathrm{SN}$ rate is in good agreement with the observed local GRB rates. It is worth noting that in the case of irregular galaxies, the predicted SN rate falls exactly in the range between the "typical" local GRB rate and its lower limit, which is consistent with the latest observations of GRB-SN hosts (e.g., Conselice et al. 2005; Savaglio et al. 2006) and with a recent work by Fruchter et al. 2006, who showed that the host galaxies of GRBs appear to be significantly faint and most of them are irregular. 
Table 1.

\begin{tabular}{lclc}
\hline \hline \multicolumn{2}{c}{ Local GRB rates $R_{\mathrm{obs}}$} & \multicolumn{2}{c}{ GRB beaming factor } \\
\hline Author & {$\left[\mathrm{GRB} \mathrm{yr}^{-1}\right]$} & Author & $\left\langle f_{\mathrm{b}}^{-1}\right\rangle$ \\
\hline Sokolov (2001) & $\sim 5.0 \times 10^{-8}$ & Schmidt et al. (2001) & $\sim 100$ \\
Zhang \& Mészáros (2004) & $\sim 4.0 \times 10^{-7}$ & Frail et al. (2001) & $520 \pm 85$ \\
Firmani et al. (2004) & $\sim 2.0 \times 10^{-7}$ & Guetta et al. (2005) & $75 \pm 25$ \\
Della Valle (2005) & $\sim 3.8 \times 10^{-7}$ & Yonestoku et al. (2005) & $\sim 340$ \\
Le \& Dermer (2006) & $\sim 1.5-1.9 \times 10^{-6}$ & Soderberg (2005) & $13 \lesssim\left\langle f_{\mathrm{b}}^{-1}\right\rangle \lesssim 10^{4}$ \\
\hline
\end{tabular}
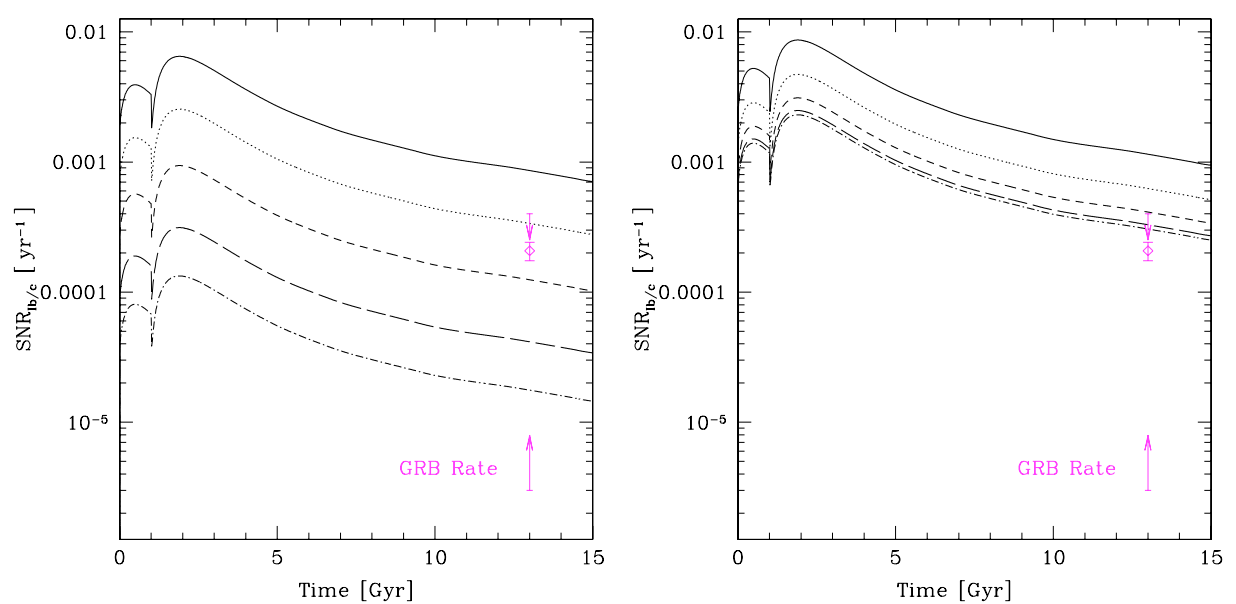

Fig. 6. SN rate calculated for model I (left panel) and model III (right panel) in a spiral galaxy for five different mass ranges of existence of type $\mathrm{Ib} / \mathrm{c}$ SNe progenitors (single WR stars): $\Delta_{\mathrm{M} 1}=25-100 M_{\odot}$ (solid line); $\Delta_{\mathrm{M} 2}=40-100 M_{\odot}$ (dotted line); $\Delta_{\mathrm{M} 3}=60-100 M_{\odot}$ (short-dashed line); $\Delta_{\mathrm{M} 4}=80-100 M_{\odot}$ (log-dashed line); $\Delta_{\mathrm{M} 5}=90-100 M_{\odot}$ (dot-dashed line). A Scalo (1986) IMF and a value of $F=0.15$ are assumed. Upper and lower limits for the observed local GRB rates (Eq. (16)) are over-plotted (upper and lower arrows). The open rhombus represents a "typical" value calculated from a local uncorrected rate by Zhang \& Mészáros (2004) and a beaming correction value by Frail (2001) (see Eq. (17)).

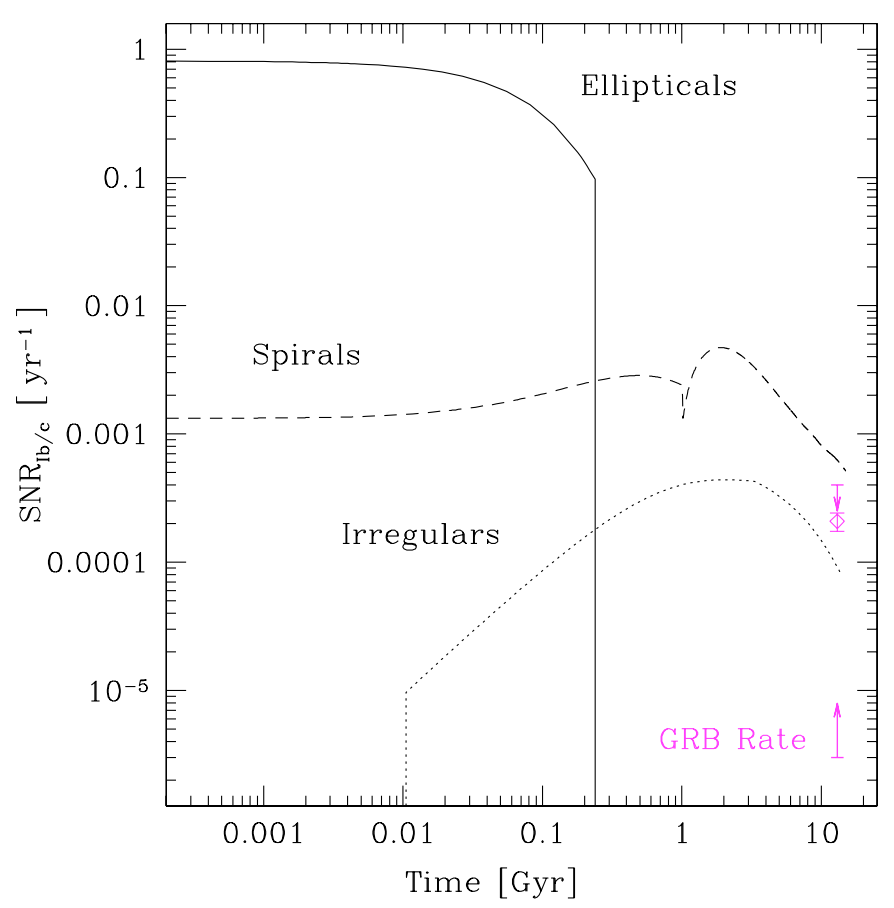

Fig. 7. Upper and lower limits for the observed local GRB rates (upper and lower arrows) compared with model III SN rate model for galaxies of different morphological types: ellipticals (solid line), spirals (dashed line) and irregulars (dotted line). The open rhombus represents a "typical" value calculated from a local uncorrected rate by Zhang \& Mészáros (2004) and a beaming correction value by Frail et al. (2001) (see Eq. (17)).
Table 2. Values of the coefficients for $\psi_{\mathrm{MDP}_{1}}(t), \psi_{\mathrm{MDP}_{2}}(t)$ and $\psi_{\mathrm{ST} 04}(t)$.

\begin{tabular}{ccccccc}
\hline \hline SFR & $\mathrm{a}$ & $\mathrm{b}$ & $\mathrm{c}$ & $\mathrm{d}$ & $\mathrm{e}$ & $\mathrm{d}$ \\
\hline$\dot{\rho}_{* \mathrm{MDP}_{1}}(t)$ & 0.049 & 5 & 0.64 & 0.2 & - & - \\
$\dot{\rho}_{* \mathrm{MDP}_{2}}(t)$ & 0.336 & 5 & 0.64 & 0.0074 & 0.0197 & 1.6 \\
$\dot{\rho}_{* \mathrm{ST} 04}(t)$ & 0.182 & 1.260 & 1.865 & 0.071 & - & - \\
\hline
\end{tabular}

\section{Cosmic $S N \mathrm{Ib} / \mathrm{c}$ and $\mathrm{GRB}$ rates and $\mathrm{GRB} / \mathrm{SNe}$ ratios}

The comoving rate density is a measure of the number of events occurring per unit comoving volume and time which provides a census of the number of objects formed at a given redshift and which can help understanding the object/structure formation in its various stages of evolution. In the following sections this quantity will be estimated and discussed for both type $\mathrm{Ib} / \mathrm{c} \mathrm{SNe}$ and GRBs.

\subsection{The cosmic $S N \mathrm{Ib} / \mathrm{c}$ rate}

In order to reproduce cosmic $\mathrm{SN} \mathrm{Ib} / \mathrm{c}$ rate densities, the fundamental ingredients are a cosmic SFR density and a suitable IMF. There are different models which can describe the global SFR per unit comoving volume. In the following, four models will be reviewed and analysed. 


\subsubsection{Cosmic SFR density models}

One model was computed by Calura (2003, hereafter CM03). They calculated the cosmic SFR density $\dot{\rho}_{\star}$ as a function of redshift (Madau's plot) in the case of a $\Lambda$ CDM cosmology and galaxy formation at $z_{\mathrm{f}}=5$. Since the cosmic SFR density is not a directly observable quantity, $\mathrm{CM} 03$ evaluated it by calculating the luminosity density (LD), $\rho_{\lambda}$, at certain wavelengths and by adopting a universal IMF (the Salpeter one).

The total LD in a given band is the integrated light radiated per unit volume from the entire galaxy population and it needs the luminosity function (LF) to be determined. The LF represents the distribution of absolute magnitudes for galaxies of any specified Hubble type and it is often parametrized according to the form defined by Schechter (1976):

$\Phi(L) \frac{\mathrm{d} L}{L_{\star}}=\Phi_{\star}\left(\frac{L}{L_{\star}}\right)^{-\gamma} \exp \left(-\frac{L}{L_{\star}}\right) \frac{\mathrm{d} L}{L_{\star}}$,

where $\Phi_{\star}$ is a normalization constant related to the number of luminous galaxies per unit volume, $L_{\star}$ is a characteristic luminosity and $\gamma$ is the slope of the luminosity function. The LD stems from the integral over all magnitudes of the observed LF:

$\rho_{L}=\int \Phi\left(L / L_{\star}\right) L / L_{\star} \mathrm{d}\left(L / L_{\star}\right)$.

The determination of the SFR density is related to the measures of star formation in galaxies. CM03 reconstructed the history of cosmic star formation in the Universe by means of detailed chemical evolution models for galaxies of different morphological types, as described before. They normalized the galaxy populations to the $B$-band LF observed in the local Universe and studied the redshift evolution of the LD in various bands $(U$, $B, I$ and $K$ ), calculating galaxy colors and evolutionary corrections by means of a detailed synthetic stellar population model (Jimenez et al. 1998). The cosmic SFR density was computed according to:

$\dot{\rho}_{\star}(z)=\sum_{i} \rho_{B, i}(z)\left(\frac{M}{L}\right)_{B, i} \psi_{i}(z)$,

where $\rho_{B, i}$ represents the $B$-band LD, $\left(\frac{M}{L}\right)_{B, i}$ is the $B$-band massto-light ratio, and $\psi_{i}$ represents the star formation rate for the galaxies of the $i$ th morphological type.

An analytical fit which reproduces the total cosmic SFR density as a function of time (CM03), is given by:

$y(t)=\left(0.337-9.37 \cdot t+11.19 \cdot t^{2}\right)$

for $\mathrm{t}<0.328 \mathrm{Gyr}$;

$y(t)=\left(-1.57+0.11 \cdot t+0.05 \cdot t^{2}\right)$

for $0.328<t<2.150 \mathrm{Gyr}$;

$y(t)=\left(-0.836-0.13 \cdot t+0.005 \cdot t^{2}\right)$

for $t>2.150 \mathrm{Gyr}$, where $y(t)=\dot{\rho}_{*}$.

The SFR density for this model is then calculated as:

$\dot{\rho}_{\mathrm{CM} 03}(t)=10^{y(t)} M_{\odot} \mathrm{yr}^{-1} \mathrm{Mpc}^{-3}$.

The CM03 model predicts a peak at the redshift of galaxy formation due to starbursts in spheroids. This peak is clearly visible at $z \sim 5$ (Fig. 8 of CM03) and it is followed by a flat behaviour between $z \sim 4.2$ and $z \sim 3$ due to star formation in spiral galaxies. The maximum star formation in spirals causes a smaller peak at $z=2$; these galaxies are also responsible for the decline of the SFR density between $z=2$ and $z=0$, in agreement with observational data.

Two other cosmic SFR density models considered for this analysis were calculated following the work done by Madau et al. (1998b), in which the emission history of field galaxies is modeled at UV, optical and near-IR wavelenghts by tracing the evolution with cosmic time of their luminosity density,

$\rho_{v}(z)=\int_{0}^{\infty} L_{v} y_{v}\left(L_{v}, z\right) \mathrm{d} L_{v}$

where $y\left(L_{v}, z\right)$ is the best-fitting Schechter luminosity function in each redshift bin. The integrated light radiated per unit volume from the entire galaxy population is an average over cosmic time of the stochastic, possibly short-lived star formation episodes of individual galaxies, and follows a relatively simple dependence on redshift. Madau et al. (1998b) used a stellar evolution model defined by a time-dependent star formation rate per unit volume, $\psi(t)$, a universal IMF, and some amount of reddening. In such a system, the luminosity density at time $t$ is given by the convolution integral:

$\rho_{v}(t)=p_{\text {esc }} \int_{0}^{t} l_{v}\left(t^{\prime}\right) \psi\left(t-t^{\prime}\right) \mathrm{d} t^{\prime}$,

where $l_{v}\left(t^{\prime}\right)$ is the specific luminosity radiated per unit initial mass by a generation of stars with age $l_{v}\left(t^{\prime}\right), p_{\text {esc }}$ is a timeindependent term equal to the fraction of emitted photons which are not absorbed by dust and the cosmic SFR density is derived from the observed UV luminosity density.

Based on these considerations, the cosmic SFR model is given by an analytical fit developed by Madau et al. (1998a, hereafter MDP98):

$\dot{\rho}_{* \mathrm{MDP}_{1}}(t)=a\left[t_{9}^{b} \mathrm{e}^{-t_{9} / c}+d\left(1-\mathrm{e}^{-t_{9} / c}\right)\right]$,

expressed in $M_{\odot} \mathrm{yr}^{-1} \mathrm{Mpc}^{-3}$ and where $t_{9}$ is the Hubble time in Gyr, and the values for the coefficients are given in Table 2.

This cosmic SFR was adopted by MDP98 in order to predict the evolution of the observed comoving luminosity density in the "hierarchical clustering" scenario, in which elliptical galaxies form continuously from the merger of disk-bulge systems or other ellipticals and most galaxies never experience star formation rates in excess of a few solar masses per year.

A second analytical fit by MDP98 consists of a large star formation density at high redshifts and was designed to mimic the "monolithic collapse" scenario as in the CM03 model, in which spheroidal systems form early and rapidly, experiencing a bright starburst phase at high- $z$ :

$\dot{\rho}_{* \mathrm{MDP}_{2}}(t)=a \mathrm{e}^{-t_{9} / f}+d\left(1-\mathrm{e}^{-t_{9} / c}\right)+\mathrm{e} t_{9}^{b} \mathrm{e}^{-t_{9} / c}$,

expressed in units of $M_{\odot} \mathrm{yr}^{-1} \mathrm{Mpc}^{-3}$, where the values of the coefficients are given in Table 2 .

The last model which will be considered in the following analysis was computed by Stolger et al. (2004, hereafter ST04), assuming a modified version of the parametric form of $\psi(t)$ as suggested by Madau et al. (1998a). An analytical expression for this model is given by:

$\dot{\rho}_{* \mathrm{ST} 04}(t)=a\left(t^{b} \mathrm{e}^{-t / c}+d \mathrm{e}^{\left(t-t_{0}\right) / c}\right)$,

always in units of $M_{\odot} \mathrm{yr}^{-1} \mathrm{Mpc}^{-3}$, where $t$ is in Gyr. By fitting the measurements of SFR from several surveys together 

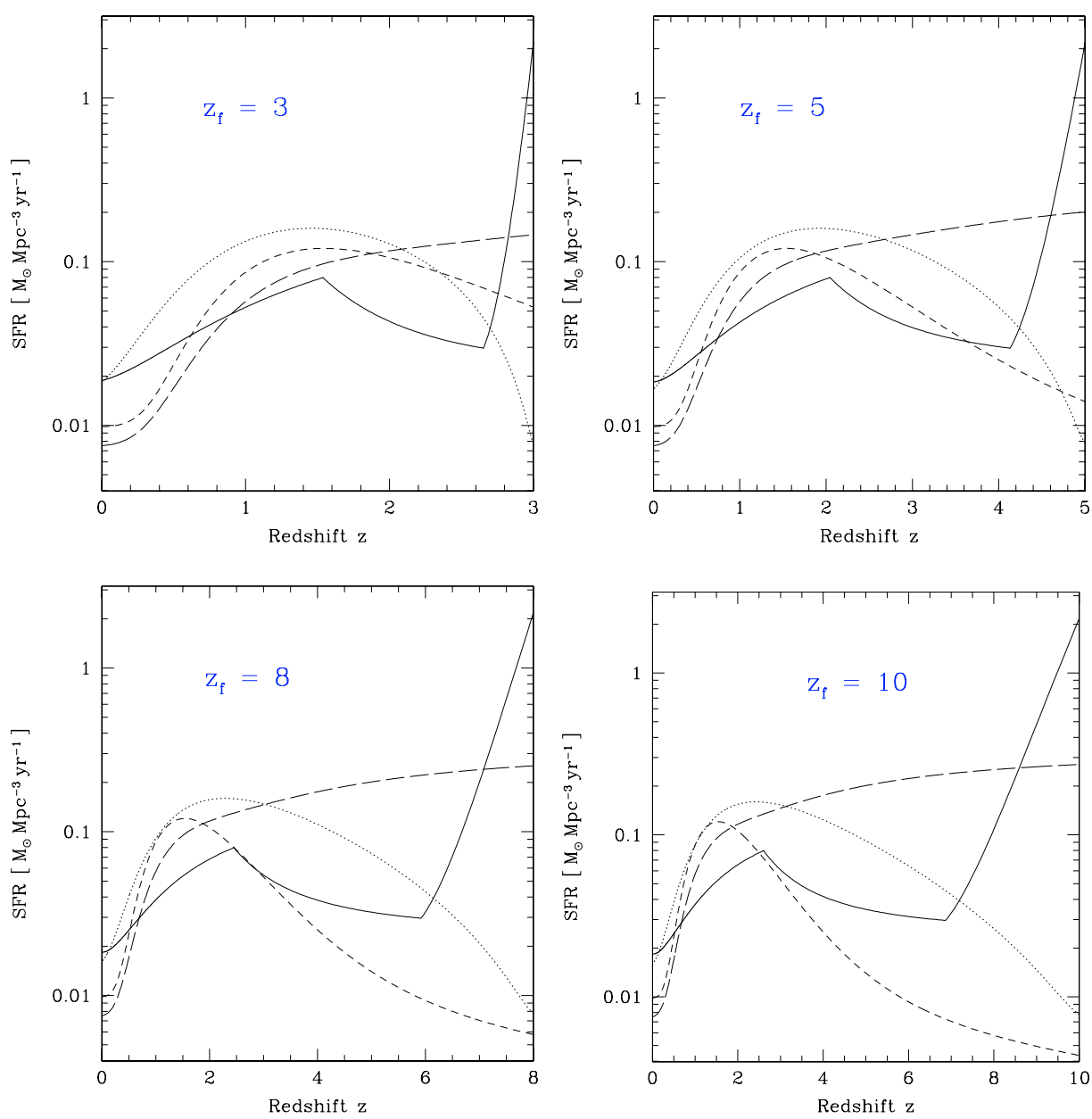

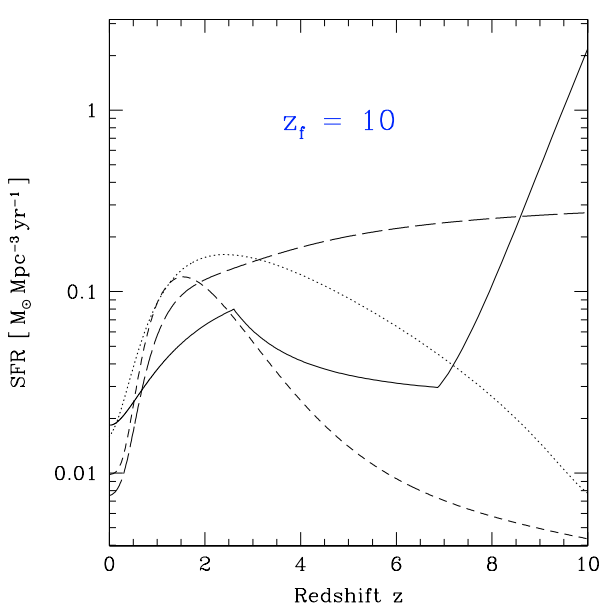

Fig. 8. Cosmic SFR densities as a function of redshift calculated for different epochs of galaxy formation, namely $z_{f}=3$ (left panel) and $z_{f}=5$ (right panel); model CM03 (Eq. (22)): solid line; model $\mathrm{MDP}_{1}$ (Eq. (25)): short-dashed line; model $\mathrm{MDP}_{2}$ (Eq. (26)): long-dashed line; model ST03 (Eq. (27)): dotted line.
(Giavalisco et al. 2004), ST determined the coefficients of the function as summarized in Table 2. This model takes the corrections for extinction into account. Here $t$ is the age of the Universe and $t_{0}=13.47$ Gyr corresponds to $z=0$.

All the already described models are plotted together as functions of redshift in Figs. 8 and 9 assuming four different epochs of galaxy formation $z_{\mathrm{f}}$, namely $3,5,8$ and 10 . The different behaviours of the cosmic SFR following the "monolithic collapse" scenario (models CM03 and $\mathrm{MDP}_{2}$ ) or the "hierarchical clustering" scenario (model MDP 1 and ST04) are clearly visible toward higher redshifts.

\subsubsection{Cosmic $\mathrm{SN}_{\mathrm{lb} / \mathrm{c}}$ models}

The strategy for estimating the cosmic SN rate is the same as the one adopted for the local case in Sect. 1.1. Equations (11), (12), and (14) for models I-III, respectively, can be calculated considering different cosmic SFRs, IMFs, and various mass ranges. From now on, the analysis will focus on the cosmic SN rate calculated for model III, i.e. taking both massive single stars and massive binary stars into account, with a SFR by CM03. This is in fact the model which gives the best fit to the assumed type Ib/c SN rates in galaxies (Sect. 1.1). Moreover, the SFR will be computed assuming galaxy formation was taking place at $z_{\mathrm{f}}=10$.

This choice of $z_{\mathrm{f}}$ is motivated by the observation of the most distant quasars (Staguhn et al. 2005), suggesting massive starbursts up to $z \sim 5$, together with an interesting result by Mobasher et al. (2005). Searching for high-redshift $J$-band "dropout" galaxies in the portion of the Great Observatories
Origins Deep Survey (GOODS) southern field that is covered by extremely deep imaging from the Hubble Ultradeep Field (HUDF), they found evidence for a massive galaxy, HUDFJD2, whose spectral energy distribution shows distinctive features which are consistent with those of a galaxy at $z \sim 6.5$, observed several hundred million years after a powerful burst of star formation. The best-fitting models adopted by Mobasher et al. placed the formation of the bulk of the stars at $z>9$. For completeness, they also reported alternative models of dusty galaxies at $z \approx 2.5$. However, these models provided significantly poorer fits to the photometric data. Mobasher et al. suggested that, if the high-redshift interpretation is correct, HUDFJD2 is an example of a galaxy that formed by a process strongly resembling traditional models of "monolithic collapse".

An interesting test is to model the $\mathrm{SN}$ rate for different IMFs. Figure 10 shows its behaviour as a function of the redshift for various choices of the IMF, namely a Salpeter IMF, a "topheavy" IMF, a Tinsley (1980) IMF, a Scalo (1986) IMF, and a Scalo (1998) IMF. The Salpeter IMF, which lies at an intermediate position among the different models, is chosen as the best IMF for performing further analysis.

\subsection{The cosmic GRB rate}

The effort to find a redshift estimate for bursts without optical afterglows represents an open research branch in the GRB cosmology field. The redshift distribution, together with the LF, can provide important insights not only into the physics of the 


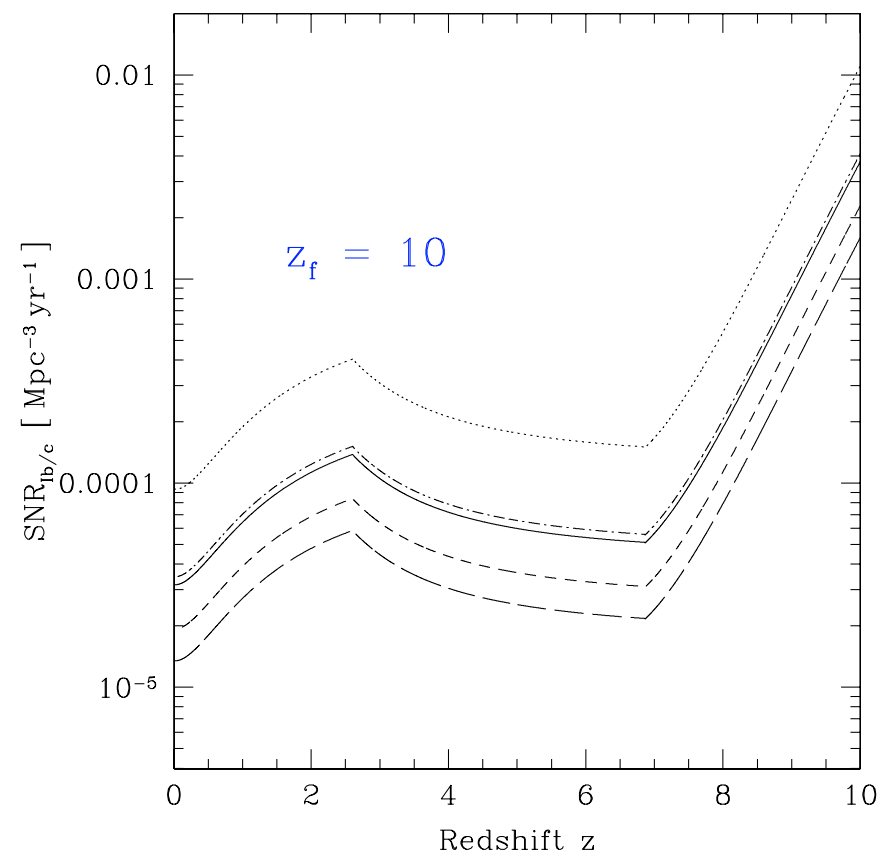

Fig. 10. Cosmic SN rate for model III based on the cosmic SFR by CM03 and different IMFs: Salpeter (1955) (Eq. (7), solid line); "topheavy" (Eq. (10), dotted line); Tinsley (1980) (short-dashed line); Scalo (1986) (Eq. (9), long-dashed line); Scalo (1998) (dot-dashed line). Redshift of galaxy formation: $z_{\mathrm{f}}=10$.

individual objects themselves, but also into the evolution of the matter in the Universe.

Often, when doing large statistical studies of a particular class of objects, the LF and redshift distribution are assumed to be independent quantities; that is, the luminosity function of the sources are assumed to be the same for all redshifts. This makes the analysis easier when one has limited information (e.g. significant data selection effects) obscuring a direct interpretation of the measured distributions of luminosity and redshift. However, it has been shown that this assumption is not valid for many astrophysical objects, i.e. quasars (Boyle 1993) and GRBs.

By studying the prompt and especially the afterglow emission in great detail, the GRB community realized from the very first bursts with measured redshifts that the GRB LF was not very narrow but in fact exhibited a rather large dispersion, hence preventing the flux of a GRB beeing used as a standard candle from which to infer a redshift for the source.

In the last few years, various types of "standard-candle relationships" for GRBs have been discussed, from which a redshift can be inferred based on common GRB observables. For example, Lloyd-Ronning et al. (2002, hereafter LFR02) studied GRB formation rates as the result of a redshift distribution estimated by Fenimore \& Ramirez-Ruiz (2000), by means of a "Variability-Luminosity" correlation. Using a $\tau$ statistical method (Lynden-Bell 1971; Efron \& Petrosian 1992), LFR02 obtained a GRB comoving rate density as:

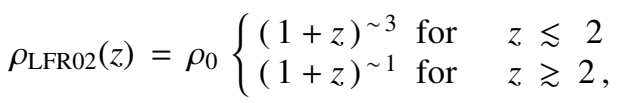

where $\rho_{0}$ is the normalization constant. This distribution was computed in units of $\mathrm{Mpc}^{-3} \mathrm{yr}^{-1}$ with an arbitrary normalization of the curve to $\sim 1$ at $(1+z)=1$.

Another attempt in the GRB redshift estimate was done by Yonetoku et al. (2004, hereafter Y04), adopting a "Peak
Luminosity-Peak Energy" correlation. Introducing the same statistical method as LFR02, they produced the following GRB rate density:

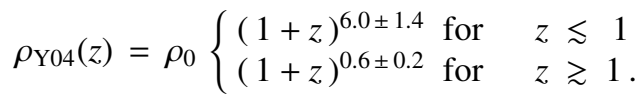

In a recent paper by Matsubayashi et al. (2005, hereafter M05), the apparent cosmic GRB rates by LFR02 and Y04 were studied and used to derive the true absolute GRB formation rate. The peculiarity of this work lies in the fact that M05 took into account the geometrical correction of the jet opening angle, $\theta_{\mathrm{j}}$, and the jet-luminosity evolution, finally presenting an analytical formula to calculate the true GRB rate.

M05 adopted the cosmic GRB formation rate proposed by Y04, performing the normalization of $\rho_{\mathrm{Y} 04}(z)$ at $z=1$, and obtained a true comoving GRB rate density given by:

$\rho_{\mathrm{M} 05}(z)=\rho_{\mathrm{Y} 04}(z)\left\langle f_{\mathrm{b}}^{-1}\right\rangle_{z}$.

They derived the distribution of the beaming factor $\left\langle f_{\mathrm{b}}^{-1}\right\rangle_{z}$ in a very detailed way, obtaining:

$\left\langle f_{\mathrm{b}}^{-1}\right\rangle_{z}=200\left(\frac{1+z}{2}\right)^{\alpha-\beta}$.

Here $\alpha$ and $\beta$ are the slope indexes of the GRB luminosity evolution $\lambda(z)$ (see LFR02 and Y04 for a detailed description),

$\lambda(z) \propto(1+z)^{\alpha}$,

and the jet-corrected luminosity evolution $L_{j}(z)$ (Lamb et al. 2005),

$L_{j}(z) \propto(1+z)^{\beta}$

respectively. M05 imposed $\left\langle f_{\mathrm{b}}^{-1}\right\rangle_{z}=200$ at $z=1$. In this case, the mean jet opening half-angle, defined by $\left\langle\theta_{\mathrm{j}}\right\rangle_{z} \equiv$ $\left[\left\langle f_{\mathrm{b}}^{-1}\right\rangle / 2\right]^{-1 / 2}$, is equal to the typical value of $0.1 \mathrm{rad}$ at $z=1$.

The value of $\alpha$ has been studied by many authors. LFR02 found $\alpha \simeq 1.4 \pm 0.5$, while Y04 calculated $\alpha \simeq 2.60_{-0.20}^{+0.15}$, which is quite similar to that of quasars. Firmani et al. (2004) found evidence supporting an evolving LF where the luminosity scales as $\alpha \simeq 1.0 \pm 0.2$. M05 adopted the value by Y04, i.e. $\alpha=2.6$, in his work.

Studying possible values for $\beta$, M05 considered three cases: a) $\beta=\alpha$ - no jet-opening angle evolution; b) $\beta=\alpha / 2-$ intermediate; c) $\beta=0-$ no jet-luminosity evolution. M05 rejected cases (a) and (c) (see the original work for an exhaustive description), concluding that intermediate values of $\beta$ in the neighbourhood of $\beta \simeq \alpha / 2$, for which both $L_{\mathrm{j}}$ and $\left\langle f_{\mathrm{b}}^{-1}\right\rangle$ depend on $z$, may be preferable. Applying a generalized $\tau$ statistical method, M05 calculated $\beta_{\mathrm{M} 05}=2.05_{-0.75}^{+0.53}$. Recently, the existence of opening angle evolution of the form $\propto(1+z)^{\gamma}$ with $\gamma=-0.45_{-0.18}^{+0.20}$ was suggested by Yonetoku et al. (2005). Combining this with the result of $\alpha$ by Y04, a value of $\beta_{Y 04}=\alpha+2 \gamma \simeq 1.7_{-0.41}^{+0.43}$ is obtained.

\subsection{Cosmic $S N_{\mathrm{lb} / \mathrm{c}}$ vs. GRB rates}

Summing up all the results gathered up to this point, a comparison between the cosmic SN rate models and the cosmic GRB rate models is the last important step to make in order to obtain a complete overview. As explained in the previous sections, 
the cosmic SN rate can be calculated in different scenarios and for different values of the parameters. Here, the cosmic SFR by CM03 is considered ("monolithic collapse scenario"), calculated for a redshift of galaxy formation $z_{f}=10$, and in units of $M_{\odot} \mathrm{Gpc}^{-3} \mathrm{yr}^{-1}$. Two different IMFs are assumed, namely the Salpeter IMF and the "top-heavy" IMF (see Sect. 1.0.3). Model III is computed assuming the usual mass range $\Delta_{\mathrm{M} 1}=$ 25-100 $M_{\odot}$ and a massive binary parameter $F=0.15$, as previously discussed.

These $\mathrm{SN}$ rate models are compared to the cosmic GRB rate density models by LFR02, Y04, and M05. Models by LFR02 and Y04 need to be normalized to the observed local GRB rate, this time expressed in units of $\mathrm{Gpc}^{-3} \mathrm{yr}^{-1}$. A typical value which is found in the literature is $\rho_{0}=\rho(z=0)=0.5 \mathrm{Gpc}^{-3} \mathrm{yr}^{-1}$ (Schmidt 2001). Taking different possible beaming corrections into account (see Table 1), a range for the true local GRB rate is obtained:

$$
\begin{aligned}
& \rho_{0, \min } \sim 38 \pm 13 \mathrm{Gpc}^{-3} \mathrm{yr}^{-1} \\
& \rho_{0, \max } \sim 260 \pm 40 \mathrm{Gpc}^{-3} \mathrm{yr}^{-1} .
\end{aligned}
$$

Figure 11 shows the behaviours of the cosmic SN rate (upper solid and dashed curves, for models by CM03 and ST04, respectively) and the estimated comoving GRB rate densities (shaded areas for models by LFR02 and Y04, and lower solid line for the model by M05). The SN rate in the left and right panels are calculated assuming a Salpeter and a "top-heavy" IMF, respectively. The GRB models by LFR02 $\left(\rho_{\text {LFR } 02}(z)\right)$ and by Y04 $\left(\rho_{\mathrm{Y} 04}(z)\right)$ are normalized in the interval between $\rho_{0 \text {, min }}$ and $\rho_{0, \max }$, corresponding to the dark grey and light grey areas, respectively. The GRB model by M05 $\left(\rho_{\mathrm{M} 05}(z)\right.$, lower solidline) is calculated according to the original parameters $\alpha=2.6$, $\beta=1.7$ and $\rho(0) \simeq 0.01 \mathrm{Gpc}^{-3} \mathrm{yr}^{-1}$.

The predicted cosmic SN rates are by far larger with respect to the estimated cosmic GRB rate models. However, this fact is not surprising. First, local GRB rates suffer from great uncertainties, mainly due to the beaming corrections. Moreover, the models applied for the cosmic GRB rate densities rely upon luminosity indicators and consequent redshift estimates, which are still far from being confirmed as "true" standard-candle relationships. It is worth noting that Fig. 11 shows that the cosmic SN Ib/c rate obtained by means of the hierarchical scenario (model ST04) predicts a much lower GRB rate at high redshift than the one predicted by the monolithic scenario (model CM03).

Looking again at Fig. 11, one can see that this ratio GRB/SNe is almost $\sim 10^{-2} \div 10^{-3}$, depending on the choice of the different parameters, which play an important role in the calculation of the $\mathrm{SN}$ rate. Table 3 summarizes in four sub-tables (A to D) all the different analyses which have been performed in this paper, giving an estimate of the local ratio between GRB and type $\mathrm{Ib} / \mathrm{c} \mathrm{SNe}$ according to the various applied models and parameters. In these subtables, two ratios are presented, namely:

$R_{\mathrm{A}}=\frac{\rho_{0, \max }}{S N R_{\mathrm{Ib} / \mathrm{c}}}(z=0)$
$R_{\mathrm{B}}=\frac{\rho_{0, \min }}{S N R_{\mathrm{Ib} / \mathrm{c}}}(z=0)$,

i.e. the ratios are calculated assuming the largest and the smallest $\left\langle f_{\mathrm{b}}^{-1}\right\rangle$. This is to underline the great uncertainty of the local GRB rate due to the beaming correction. TABLE A displays models IIII with the usual choice of the parameters, i.e. $\Delta_{\mathrm{M}_{1}}=25-$ $100 M_{\odot}, F=0.15$ and a Salpeter IMF. Model III is our best model. $T A B L E B$ focuses on the $\mathrm{SN}$ rate calculated for the massive single star model, model I, assuming 4 different mass ranges $\left(\Delta_{\mathrm{M}_{2}}-\Delta_{\mathrm{M}_{5}}\right)$ and a Salpeter IMF, while TABLE $C$ highlights the $\mathrm{SN}$ rate calculated for the massive binary star model, model II, calculated for 5 different values of the binary parameter $F$ and for a Salpeter IMF. Finally, TABLE D displays the cumulative model, Model III, calculated assuming the usual parameters, $\Delta_{\mathrm{M}_{1}}$ and $F=0.15$, but with different assumptions for the IMF. Still, the results are more or less consistent with previous works (Podsiadlowski et al. 2004; Della Valle 2005; Le \& Dermer 2006).

While the scatter of possible values for the GRB-SN ratio is mainly due to uncertainties affecting the observed local GRB rate and the beaming factor, the ratio is intrinsically small. This is caused by the fact that only a very tiny fraction of all massive stars are capable of producing GRBs. Type Ib/c SN represent "obvious" candidates because they lose the H envelope when the collapse of the core occurs, therefore allowing the ultrarelativistic jets to escape from the massive progenitor. However this fact does not seem to be sufficient. According to the SN and GRB rates and to the $\left\langle f_{\mathrm{b}}^{-1}\right\rangle$ estimates, only $1 \%-0.1 \%$ of $\mathrm{SNe}_{\mathrm{Ib} / \mathrm{c}}$ are able to produce GRBs. This fact implies that GRB progenitors must have some other special characteristic other than just being massive stars. Recently, several authors have discussed a number of promising mechanisms at play in the GRB phenomenon, such as stellar rotation (Woosley \& Heger 2006), binarity (Podsiadlowski et al. 2004; Mirabel 2004), asymmetric explosions (Maeda et al. 2006), and metallicity (Fruchter et al. 2006; Langer \& Norman 2006).

\section{Conclusions}

In this paper we have compared the local GRB rate with local type $\mathrm{Ib} / \mathrm{c} \mathrm{SN}$ rates in galaxies of all morphological types: ellipticals, spirals, and irregulars. To do that we have adopted for each galaxy type a specific history of star formation already tested in self-consistent models of galactic chemical evolution, which reproduce the main chemical and photometric properties of galaxies and in particular the observed SN rates. We have assumed that type $\mathrm{Ib} / \mathrm{c} \mathrm{SNe}$, which are associated to longduration, soft GBRs, originate either from massive single stars ( $M>25 M_{\odot}$, WR stars) or from massive stars in the range 12$20 M_{\odot}$, which are members of close binary systems. We did several tests by varying the IMF and the mass range for the existence of WR stars. This decision/test is justified by the fact that uncertainties still exist regarding the progenitors of WR stars.

Moreover, we computed the cosmic star formation rate in a comoving unitary volume of the Universe by taking into account all the galactic types and adopting a technique developed by CM03. Then the cosmic star formation rate was used to compute the cosmic type Ib/c SN rate as a function of redshift. In this particular formulation, the only one that distinguishes among different galactic morphological types, we assumed that elliptical galaxies, especially the most massive ones, form quickly and at high redshift, whereas the spirals and irregulars evolve much more slowly. The main driver of galaxy evolution is assumed to be the star formation rate, which is very fast in ellipticals and spheroids in general, and slower in spirals and irregulars. This scenario is different from the classic hierarchical scenario of galaxy formation, where ellipticals are assumed to have assembled through mergers and preferentially at low redshift. Because of this, we also tested different cosmic star formation rates and type $\mathrm{Ib} / \mathrm{c} \mathrm{SN}$ rates including the hierarchical scenario. We then 

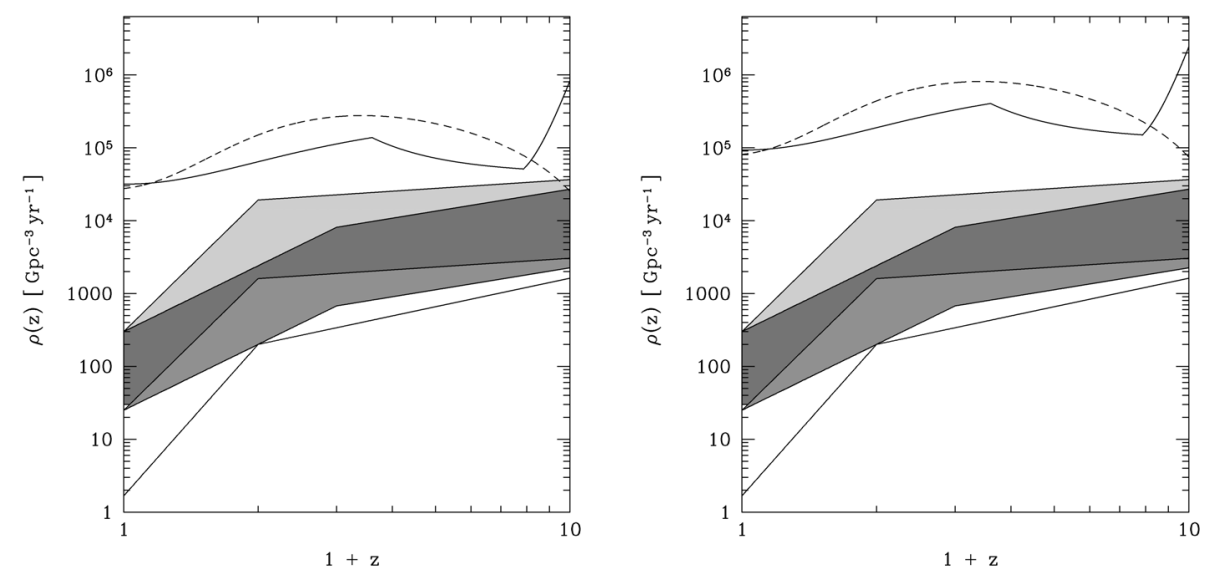

Fig. 11. Absolute cosmic SN rate for model III calculated for the SFR by CM03 (upper solid curve) and by ST04 (dashed curve), assuming first a Salpeter IMF (Eq. (7), left panel) and then a "top-heavy" IMF (Eq. (10), right panel). The plots show also three absolute comoving GRB rate densities, calculated according to the models by LFR02 $\left(\rho_{\mathrm{LFR} 02}(z)\right.$, Eq. (28)), Y04 ( $\rho_{\mathrm{Y} 04}(z)$, Eq. (29)) and M05 $\left(\rho_{\mathrm{M} 05}(z)\right.$, Eq. (30), lower solid line). Models by LFR02 and Y04 are normalized in an interval between two local GRB rates, $\rho_{0 \text {, min }}$ (bottom lines) and $\rho_{0, \max }$ (upper lines, see Eq. (34)), covering two shaded areas of possible values (light grey, superior and dark grey, inferior areas, respectively).

Table 3. GRB/SN ratios $R_{A}$ and $R_{B}$ (Eqs. (35)) calculated according to the three models previously discussed (see (Eqs. (11), (12) and (14)). The ratios are calculated for models I to III with the usual choice of the parameters $\left(\Delta_{\mathrm{M}_{1}}=25-100 M_{\odot}, F=0.15\right.$ and a Salpeter IMF, TABLE A) and then for each model tuned on different parameters: TABLE B displays model I, assuming 4 different mass ranges $\left(\Delta_{\mathrm{M}_{2}}-\Delta_{\mathrm{M}_{5}}\right)$ and a Salpeter IMF; TABLE $C$ highlights model II, calculated for 5 different values of the binary parameter $F$ and for a Salpeter IMF; TABLE $D$ displays model III, calculated assuming the usual parameters, $\Delta_{\mathrm{M}_{1}}$ and $F=0.15$, but different assumptions for the IMF.

\begin{tabular}{|c|c|c|}
\hline \multicolumn{3}{|c|}{ TABLE A } \\
\hline & $R_{\mathrm{A}}$ & $R_{\mathrm{B}}$ \\
\hline Model I ${ }^{a, c}$ & $\sim 1.2 \times 10^{-2}$ & $\sim 9.7 \times 10^{-4}$ \\
\hline Model $\mathrm{II}^{b, c}$ & $\sim 4.9 \times 10^{-2}$ & $\sim 4.1 \times 10^{-3}$ \\
\hline Model III ${ }^{a, b, c}$ & $\sim 9.4 \times 10^{-3}$ & $\sim 7.9 \times 10^{-4}$ \\
\hline \multicolumn{3}{|c|}{ TABLE B } \\
\hline Model I $^{c}$ & $R_{\mathrm{A}}$ & $R_{\mathrm{B}}$ \\
\hline$\Delta_{\mathrm{M}_{2}}=40-100 M_{\odot}$ & $\sim 2.6 \times 10^{-2}$ & $\sim 2.2 \times 10^{-3}$ \\
\hline$\Delta_{\mathrm{M}_{3}}=60-100 M_{\odot}$ & $\sim 6.5 \times 10^{-2}$ & $\sim 5.4 \times 10^{-3}$ \\
\hline$\Delta_{\mathrm{M}_{4}}=80-100 M_{\odot}$ & $\sim 1.8 \times 10^{-1}$ & $\sim 1.5 \times 10^{-2}$ \\
\hline$\Delta_{\mathrm{M}_{5}}=90-100 M_{\odot}$ & $\sim 4.2 \times 10^{-1}$ & $\sim 3.5 \times 10^{-2}$ \\
\hline \multicolumn{3}{|c|}{ TABLE C } \\
\hline Model II ${ }^{c}$ & $R_{\mathrm{A}}$ & $R_{\mathrm{B}}$ \\
\hline$F=0.1$ & $\sim 7.4 \times 10^{-2}$ & $\sim 6.1 \times 10^{-3}$ \\
\hline$F=0.2$ & $\sim 3.7 \times 10^{-2}$ & $\sim 3.1 \times 10^{-3}$ \\
\hline$F=0.3$ & $\sim 2.5 \times 10^{-2}$ & $\sim 2.0 \times 10^{-3}$ \\
\hline$F=0.4$ & $\sim 1.8 \times 10^{-2}$ & $\sim 1.5 \times 10^{-3}$ \\
\hline$F=0.5$ & $\sim 1.5 \times 10^{-2}$ & $\sim 1.2 \times 10^{-3}$ \\
\hline \multicolumn{3}{|c|}{ TABLE D } \\
\hline Model III ${ }^{a, b}$ & $R_{\mathrm{A}}$ & $R_{\mathrm{B}}$ \\
\hline "top-heavy" IMF & $\sim 3.2 \times 10^{-3}$ & $\sim 2.7 \times 10^{-4}$ \\
\hline Tinsley IMF & $\sim 1.6 \times 10^{-2}$ & $\sim 1.3 \times 10^{-3}$ \\
\hline Scalo 1986 IMF & $\sim 2.2 \times 10^{-2}$ & $\sim 1.9 \times 10^{-3}$ \\
\hline Scalo 1998 IMF & $\sim 8.6 \times 10^{-3}$ & $\sim 7.2 \times 10^{-4}$ \\
\hline
\end{tabular}

${ }^{a} \Delta_{\mathrm{M}_{1}}=25-100 M_{\odot} \cdot{ }^{b} F=0.15 .{ }^{c}$ Salpeter IMF.

derived the GRB rate as a function of redshift and compared it with all the cosmic type $\mathrm{Ib} / \mathrm{c} \mathrm{SN}$ rates considered.

Our main conclusions can be summarized as follows:

- The best agreement between the observed and predicted local type $\mathrm{Ib} / \mathrm{c} \mathrm{SN}$ rate in galaxies of different morphological type is obtained by assuming that type Ib/c SNe progenitors include both single massive WR stars and massive stars in binary systems, in agreement with previous results by
Calura \& Matteucci (2006). Therefore, we conclude that a model including both progenitors should be preferred to compare with the GRB rate.

- When comparing the local GRB rate with the predicted local type $\mathrm{Ib} / \mathrm{c} \mathrm{SN}$ rate, the best agreement is reached under the assumptions that WR stars originate in the mass range 40-100 $M_{\odot}$ and the predictions for a typical irregular galaxy are those which best fit the local GRB rate. This seems to confirm the recent finding by Fruchter et al. (2006) indicating 
that the hosts of GRBs are mainly irregular galaxies. Since irregular galaxies are normally metal-poor objects, this would explain the need of having WR stars only for $M>40 M_{\odot}$ as due to the generally less efficient mass loss for metal-poor stars (see Maeder 1992; Meynet \& Mader 2005; see also the most recent papers by Yoon \& Langer 2005 and Yoon et al. 2006 for the connection between WR stars and GRBs.)

- The predicted cosmic SN rate computed with the cosmic star formation rate of $\mathrm{CM} 03$ follows the derived GRB rate as a function of redshift and predicts more GRBs at high redshift than do hierarchical models. In particular, the predicted rate is larger than the cosmic GRB rate, for the whole lifetime of the Universe. This is not surprising since very likely only a small fraction of type $\mathrm{Ib} / \mathrm{c} \mathrm{SNe}$ will give rise to GRBs.

- The ratio between cosmic type $\mathrm{Ib} / \mathrm{c} \mathrm{SN}$ rate and GRB rate, as predicted by our best model, is found to be in the range of $\simeq 10^{-2}-10^{-3}$ for a large varieties of cases (different IMFs, different assumptions on type Ib/c SN progenitors). We conclude that only $\simeq 0.1-1 \%$ of $\mathrm{SNe} \mathrm{Ib} / \mathrm{c}$ are able to produce GRBs. This means that other special characteristics must play a role in creating GRBs: stellar rotation, asymmetric explosions and metallicity are all possible factors.

- Because of the high star formation rate in spheroids at high redshift assumed in our best models, we predict a higher number of GRBs at high redshift than do hierarchical models.

Acknowledgements. E. B. wishes to thank Massimo Della Valle for interesting discussions and many stimulating comments and an anonymous referee for his/her usueful suggestions.

\section{References}

Arimoto, N., \& Yoshii, Y. 1987, A\&A, 173, 23

Baron, E. 1992, MNRAS, 255, 267

Bertola, F. 1964, Ann. Astroph., 27, 319

Bloom, J. S., Kulkarni, S. R., \& Djorgovski, S. G. 2002, AJ, 123, 1111

Boyle, B. J. 1993, in The Environment and Evolution of Galaxies, ed. J. M. Shull, \& H. A. Thronson (Dordrecht: Kluwer), 433

Brown, G. E., Heger, A., Langer, N., et al. 2001, NewA, 6, 457

Calura, F., \& Matteucci F. 2003, ApJ, 596, 734

Calura, F., \& Matteucci F. 2006, ApJ, 652, 889

Campana, S., Mangano, V., Blustin, A. J., et al. 2006, Nature, 442, 1008

Cappellaro, E., Evans, R., \& Turatto, M. 1999, A\&A, 351, 459

Casertano, S., de Mello, D., Dickinson, M., et al. 2000, AJ, 120, 2747

Chiappini, C., Matteucci F., \& Gratton, R. 1997, ApJ, 477, 765

Chiappini, C., Matteucci F., \& Padoan, P. 2000, ApJ, 528, 711

Chiosi, C. 1980, A\&A, 83, 206

Chugai, N. 1997, ARep, 41, 672

Conselice, C. J., Vreeswijk, P. M., Fruchter, A. S., et al. 2005, ApJ, 633, 29

Della Valle, M. 2005, in Il Nuovo Cimento C, 28, 563

Della Valle, M., 2006, in Gamma Ray Bursts in the Swift Era, Washington, D.C., ed. S. Holt, N. Gehrels \& J. A. Nousek, AIP, 836, 367

Deng, J., Tominaga, N., Mazzali, P. A., Maeda, K., \& Nomoto, K. 2005, ApJ, 624,898

Efron, B., \& Petrosian, V. 1992, ApJ, 399, 345

Elias, J. H., Matthews, K., Neugebauer, G., \& Persson, S. E. 1985, ApJ, 296, 379

Fenimore, E. E., \& Ramirez-Ruiz, E. 2000, [arXiv:astro-ph/0004176]

Filippenko, A. V., \& Sargent, W. L. W. 1991, IAUS, 143, 529

Firmani, C., Avila-Reese, V., Ghisellini, G., \& Tutukov, A. V. 2004, ApJ, 611, 1033

Frail, D. A., Kulkarni, S. R., Sari, R., et al. 2001, Nature, 562, 55

Fruchter, A. S., Levan, A. J., Strolger, L., et al. 2006, Nature, 441, 463

Galama, T. J., Vreeswijk, P. M., van Paradijs, J., et al. 1998, Nature, 395, 670

Giavalisco, M., Dickinson, M., Ferguson, H. C., et al. 2004, ApJ, 600, 103

Guetta, D., Piran, T., \& Waxman, E. 2005, ApJ, 619, 412

Hamuy, M. 2003, in Core Collapse of Massive Stars; ed. Fryer C. (Dordrecht: Kluwer)

Hjorth, J., Sollerman, J., Møller, P., et al. 2003, Nature, 423, 847
Iwamoto, K., Mazzali, P. A., Nomoto, K., et al. 1998, Nature, 395, 672 Jeffries, R. D., \& Maxted, P. F. L. 2005, AN, 326, 944

Jimenez, R., Padoan, P., Matteucci, F., \& Heavens, A. F. 1998, MNRAS, 299, 123

Kennicutt, R. C. 1998, ApJ, 498, 541

Kent, S. M., Dame, T. M., \& Fazio G. 1991, ApJ, 378, 131

Kobulnicky, H. A., Fryer, C. L., \& Kiminki, D. C. 2006, ApJ submitted, [arXiv: astro-ph/0605069]

Kroupa, P. 2002, ASPC, 285, 86

Langer, N., \& Norman, C. A. 2006, ApJ, 638, L63

Lamb, D. Q., Donaghy, T. Q., \& Graziani C. 2005, ApJ, 620, 355

Larson, R. B. 1998, MNRAS, 301, 569

Le, C., \& Dermer, C. D. 2006, ApJ submitted, [arXiv: astro-ph/0610043]

Lloyd-Ronning, N. M., Fryer C. L., \& Ramirez-Ruiz E., 2002, ApJ, 574, 554

Lynde-Bell, D. 1971, MNRAS, 155, 95

MacFadyen, A., \& Woosley S. 1999, ApJ, 524, 262

Madau, P., Della Valle, M., \& Panagia N. 1998a, MNRAS, 297, L17

Madau, P., Pozzetti L., \& Dickinson M. 1998b, ApJ, 498, 106

Maeda, K., Mazzali P., \& Nomoto K. 2006, ApJ, in press

Maeder, A. 1992, A\&A, 264, 105

Malesani, D., Tagliaferri, G., Chincarini, G., et al. 2004, ApJ, 609, L5

Mannucci, F., Della Valle, M., Panagia, N., et al. 2005, A\&A, 433, 807

Masetti, N., Palazzi, E., Pian, E., \& Patat, F. 2006, GCN 4803

Matsubayashi, T., Yamazaki, R., Yonetoku, D., Murakami, T., \& Ebisuzaki, T. 2005, PThPh, 114, 983

Matteucci, F. 1992, ApJ, 397, 32

Matteucci, F., \& François, P. 1989, MNRAS, 239, 885

Matteucci, F., \& Tornambé, A. 1987, A\&A, 185, 51

Mazzali, P. A., Deng, J., Nomoto, K., et al. 2006a, Nature, 442, 1018

Mazzali, P. A., Deng, J., Pian, E., et al. 2006b, ApJ, 645, 1323

Meynet G. \& Maeder A. 2005, A\&A, 429, 581

Mirabel, I. A. 2004, RMxAC, 20, 14

Mobasher, B., Dickinson, M., Ferguson, H. C., et al. 2005, ApJ, 635, 832

Modjaz, M., Stanek, K. Z., Garnavich, P. M., et al. 2006, ApJ, 645, L21

Muller, R. A., Newberg, H. J. M., Pennypacker, C. R., et al. 1992, ApJ, 384, L9

Nakamura, T. 2001, ApJ, 555, 880

Nomoto, K., Iwamoto, K., Suzuki, T., et al. 1996, IAUS, 165, 119

Paczyǹski, B. 1998, ApJ, 494, L45

Podsiadlowski, Ph., Joss, P. C., \& Hsu, J. J. L. 1992, ApJ, 391, 246

Podsiadlowski, Ph., Mazzali, P. A., Nomoto, K., Lazzati, D., \& Cappellaro, E. 2004, ApJ, 607, L17

Salpeter, E. E. 1955, ApJ, 121, 161

Sari, R., Piran, T., \& Halpern, J. P. 1999, ApJ Lett., 519, L17

Sauer, D. N., Mazzali, P. A., Deng, J., et al. 2006, MNRAS, 369, 1939

Savaglio S., Glazebrook K. and Le Borgne D., 2006, In Gamma Ray Bursts in the Swift Era, Washington, D.C., ed. S. Holt, N. Gehrels \& J. A. Nousek, AIP, 836, 540

Scalo, J. M. 1986, Fund. Cosm. Phys., 11, 1

Scalo, J. M. 1998, in The Stellar Initial Mass Function, ed. G. Gilmore, \& D. Howell (San Francisco: ASP), ASP Conf. Ser., 142, 201

Schechter, P., 1976, ApJ, 203, 297

Schmidt, M., 2001, ApJ, 552, 36

Soderberg, A. M., Kulkarni, S. R., Fox, D. B., et al. 2005, ApJ, 627, 877

Sokolov, V. V. 2001, BSAO, 51, 38

Staguhn, J. G., Stern, D., Benford, D. J., et al. 2005, ApJ, 629, 633

Stanek, K. Z., Matheson, T., Garnavich, P. M., et al. 2003, ApJ, 591, L17

Strolger, L.-G., Riess, A. G., Dahlen, T., et al. 2004, ApJ, 613, 200

Tinsley, B. M. 1980, Fund. Cosm. Phys., 5, 287

van der Kruit, P. C. 1986, A\&A, 157, 230

Vangioni-Flam, E., Lequeux, J., Maucherat-Joubert, M., \& Rocca-Volmerange, B. 1980, A\&A, 90, 73

Wheeler, J. C., \& Harkness, R. P. 1986, Galaxy distances and deviations from universal expansion Proceedings of the NATO Advanced Research Workshop, Kona, HI (Dordrecht: D. Reidel Publishing Co.), 455

Wijers, R. A. M. J., Bloom, J. S., Bagla, J. S., \& Natarajan, P., et al. 1998, MNRAS, 294, L13

Woosley, S. 1993, ApJ, 405, 273

Woosley, S. E., \& Heger, A. 2006, ApJ, 637, 914

Yoon, S.-C., \& Langer, N. 2005, A\&A, 443, 643

Yoon, S.-C., Langer N., \& Norman, C. 2006, A\&A, 460, 199

Yonetoku, D., Murakami, T., Nakamura, T., et al. 2004, ApJ, 609, 935

Yonetoku, D., Yamazaki, R., Nakamura, T., \& Murakami, T., et al. 2005, MNRAS, 362, 1114

Zhang, B., Woosley, S. E., \& MacFadyen, A. I. 2003, ApJ, 586, 356

Zhang, B., \& Mészáros, P. 2004, IJMPA19, 2385

Zwicky, F. 1938, PASP, 50, 215 